\title{
Clam focal and systemic immune responses to QPX infection revealed by RNA-seq technology
}

\author{
Kailai Wang ${ }^{1}$, Carmelo del Castillo ${ }^{1}$, Erwan Corre ${ }^{2}$, Emmanuelle Pales Espinosa ${ }^{1}$ and Bassem Allam ${ }^{1 *}$
}

\begin{abstract}
Background: The hard clam Mercenaria mercenaria is an important seafood species widely exploited along the eastern coasts of the United States and play a crucial role in coastal ecology and economy. Severe hard clam mortalities have been associated with the protistan parasite QPX (Quahog Parasite Unknown). QPX infection establishes in pallial organs with the lesions typically characterized as nodules, which represent inflammatory masses formed by hemocyte infiltration and encapsulation of parasites. QPX infection is known to induce host changes on both the whole-organism level and at specific lesion areas, which imply systemic and focal defense responses, respectively. However, little is known about the molecular mechanisms underlying these alterations.

Results: RNA-seq was performed using Illumina Hiseq 2000 (641 Million 100 bp reads) to characterize M. mercenaria focal and systemic immune responses to QPX. Transcripts were assembled and the expression levels were compared between nodule and healthy tissues from infected clams, and between these and tissues from healthy clams. De novo assembly reconstructed a consensus transcriptome of 62,980 sequences that was functionallyannotated. A total of 3,131 transcripts were identified as differentially expressed in different tissues. Results allowed the identification of host immune factors implicated in the systemic and focal responses against QPX and unraveled the pathways involved in parasite neutralization. Among transcripts significantly modulated upon host-pathogen interactions, those involved in non-self recognition, signal transduction and defense response were over-represented. Alterations in pathways regulating hemocyte focal adhesion, migration and apoptosis were also demonstrated.
\end{abstract}

Conclusions: Our study is the first attempt to thoroughly characterize M. mercenaria transcriptome and identify molecular features associated with QPX infection. It is also one of the first studies contrasting focal and systemic responses to infections in invertebrates using high-throughput sequencing. Results identified the molecular signatures of clam systemic and focal defense responses, to collectively mediate immune processes such as hemocyte recruitment and local inflammation. These investigations improve our understanding of bivalve immunity and provide molecular targets for probing the biological bases of clam resistance towards QPX.

Keywords: Hard clam, Mercenaria mercenaria, QPX, RNAseq, Immune response, Focal, Systemic

\footnotetext{
* Correspondence: Bassem.Allam@stonybrook.edu

${ }^{1}$ School of Marine and Atmospheric Sciences, Stony Brook University, Stony

Brook, NY 11794-5000, USA

Full list of author information is available at the end of the article
} 


\section{Background}

The hard clam, Mercenaria mercenaria, is an ecologically- and economically-important marine bivalve species that thrives along the northeastern coasts of the United States and Maritime Canada. In the past few decades, the hard clam industry has been severely impacted by a protistan parasite called QPX (Quahog Parasite Unknown), which is responsible for mortality episodes in both wild and cultured clam populations [1-7]. QPX is believed to be an opportunistic pathogen and has been detected in a wide variety of environmental substrates including sediments, marine aggregates and other organic matrices [8-10]. Interestingly, previous reports highlight the ability of QPX to sustain very low abundance in clams without causing disease outbreaks until it encounters hosts with reduced immunity or following shifts of environmental conditions that add to the virulence of the parasite, under which conditions QPX can take advantage to establish infection sometimes leading to severe clam mortality events $[4,11]$.

Lesions caused by QPX, usually associated with the presence of nodules, are commonly found in pallial tissues, such as alongside the inner rim of the mantle or at the base of the siphon $[1,2]$. These places are widely considered as the portal of entry for QPX cells acquired from the surrounding environment during suspensionfeeding $[5,7]$. The QPX nodules represent inflammatory masses containing both parasite cells and abundant clam hemocytes, resulting from a series of comprehensive host immune responses induced by the infection that leads to massive focal hemocyte infiltration, parasite encapsulation and partial necrosis of the affected area [1]. Like other invertebrates, the hard clam lacks the specific immune responses and their defense mechanism mainly relies on the effectors of innate immunity, which is mediated by circulating hemocytes and highly diversified humoral antimicrobial factors. These cellular and humoral immune components work in a synergistic way to initiate the recognition, segregation and ultimately elimination of pathogens and other non-self entities [12, 13]. The launching of innate immune responses involves myriad cellular and humoral events modulated not only at the infection sites (focally) but also at a larger, whole-organism scale (systemically). In general, the focal response represents the alterations driven by direct host-pathogen interactions at the infection sites where direct cell-cell (e.g., molecular patterns) interactions mediate the response; while the systemic response reflects overall modifications within the host as a result of the ongoing infection and is mainly associated with dynamic changes of circulating hemocytes and their secreted immune mediators.

Most of the previous investigations have solely focused on the systemic response of $M$. mercenaria against QPX during the infection events, where changes in cellular and humoral immune parameters (e.g., anti-QPX activity and lysozyme activity in clam plasma, hemocyte phagocytic activity, reactive oxygen species (ROS) production, etc.) as well as expression of a limited number of immune-related genes in tissues and circulating hemocytes were assessed [11, 14-16]. In contrast, no previous studies have focused on the characterization of clam focal response at the infection sites. Given the fact that QPX disease is usually focal with formation of welldelimited lesions, the study of clam immune responses at the infection site in the lesions per se is of specific value as it provides insights to better characterize cellular interactions between the hard clam and QPX upon their contact. In this framework, QPX disease in clams offer a unique opportunity to contrast focal and systemic responses against microbial diseases in invertebrates allowing for a more comprehensive understanding of defense strategies used by these animals to fend microbial attacks.

Our study aimed to characterize the gene regulation features of $M$. mercenaria during QPX infection by profiling the transcripts at the infection lesion and compare focal clam responses with systemic responses detected in healthy tissues from infected clams (in addition to a parallel comparison with tissues from healthy clams). This study allowed the identification of factors involved in the interactions with the parasite as well as molecular pathways activated by the host to neutralize QPX.

\section{Results and discussion}

Illumina sequencing and de novo transcriptome assembly

The main objective of this study was to identify molecular features of $M$. mercenaria in response to QPX infection and to compare the immune-related pathways involved in the lesion-specific focal response with the whole-organism scale systemic response. The highthroughput Illumina RNA sequencing and de novo assembly employed in this investigation allowed the construction of the transcriptome in the absence of $M$. mercenaria genome information. A total of 640,596,320 of $100 \mathrm{bp}$ raw reads were generated from the Illumina paired-end sequencing with about 27 to 48 Millions paired-end reads generated from each of the 9 sequenced libraries (Table 1, Fig. 1a). The short read sequences generated from this RNAseq project have been deposited at the NCBI short Read Archive database under the SRA accession number SRP068241. Trimming and filtering procedures yielded $606,021,407$ clean reads that were used for the de novo assembly of consensus transcriptome based on all sequenced RNA libraries in order to maximize the diversity of transcripts. This allowed 90.61 to $92.20 \%$ of the reads from the 9 libraries be used for the transcriptome assembly. A total of 62,980 transcripts ranging from 201 to 23,103 bp with 
Table 1 RNA samples for RNA-seq libraries. Each pool is made with equal amounts of RNA from 3 individual clams. Pools A and B were derived from the same clams

\begin{tabular}{llllll}
\hline & Library & Clams & Clam status & Tissue status & N paired-end reads \\
\hline Nodule & A1 & $1,2,3$ & Diseased & Infection foci & $30,491,569$ \\
& A2 & $4,5,6$ & Diseased & Infection foci & $34,515,597$ \\
Non-nodule & A3 & $7,8,9$ & Diseased & Infection foci & $46,861,893$ \\
& B1 & $1,2,3$ & Diseased & Non-lesion/Healthy & $27,119,432$ \\
Healthy & B2 & $4,5,6$ & Diseased & Non-lesion/Healthy & $28,254,720$ \\
& B3 & $7,8,9$ & Diseased & Non-lesion/Healthy & $40,259,333$ \\
& C1 & $10,11,12$ & Healthy & Healthy & $36,714,347$ \\
& C2 & $13,14,15$ & Healthy & Healthy & $43,293,545$ \\
\hline
\end{tabular}

average size of $1297.59 \mathrm{bp}$ and median size of $835 \mathrm{bp}$ were produced from the assembly after low FPKM and rare isoforms filtering. The size distribution of all the de novo assembled transcripts is shown in Fig. $1 \mathrm{~b}$. Once the transcriptome was constructed, the 9 libraries were individually remapped to the 62,980 transcripts and resulted with 85.27 to $89.05 \%$ of reads remapping. Theses counting data were then used for DE analysis.

\section{Transcriptome functional annotation}

The transcriptome annotation performed using Blast2GO returned a total of 19,107 transcripts (30.3\%) with significant BlastX homology matches to other sequences in NCBI nr database (E-value < 10E-03) (Fig. 1). Not surprisingly, the top 3 species that had the most similarity to $M$. mercenaria sequences were mollusks with available genomes and included the Pacific oyster, Crassostrea gigas $(7,467)$, followed by the owl limpet Lottia

A

M. mercenaria transcriptome sequencing, assembly and annotation overview

\begin{tabular}{lllllc}
\hline Sequencing results & Assembly results & & \multicolumn{2}{c}{ Annotation results } \\
\hline Read bases (G) & 65.70 & Transcripts & 62,980 & Nr annotation & 19,107 \\
Read pairs & $320,298,160$ & Average length of transcripts (bp) & 1,298 & GO mapped & 13,584 \\
Read length (bp) & 100 & Smallest transcripts (bp) & 201 & KO mapped & 6,425 \\
& & Largest transcripts (bp) & 23,103 & InterPro hits & 29,815 \\
\hline
\end{tabular}

B Size distribution of $M$. mercenaria de novo assembled transcriptome obtained by Trinity

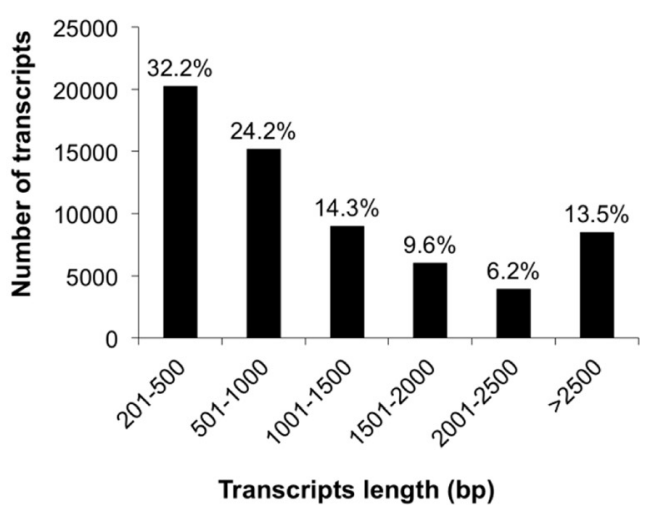

C

BLAST top hits species distribution of annotated sequences

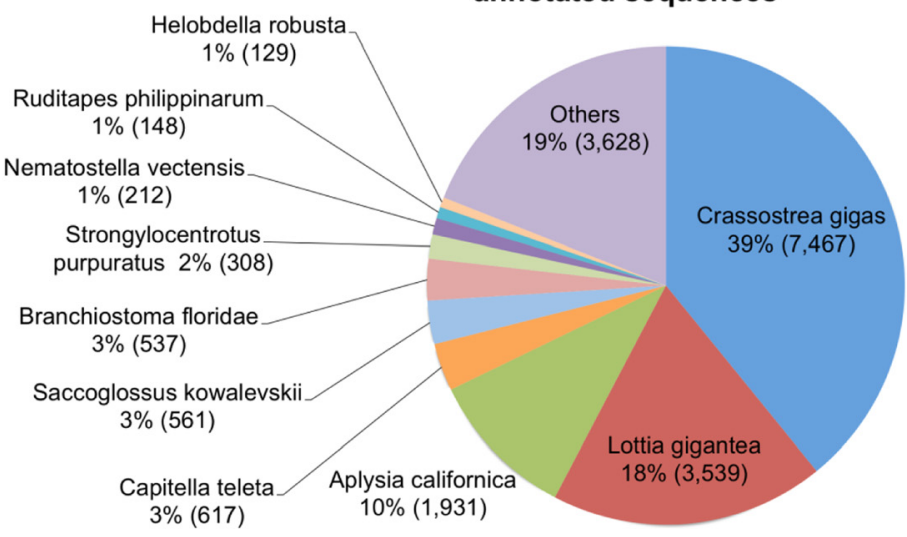

Fig. 1 M. mercenaria de novo assembled transcriptome summary. a Transcriptome sequencing, assembly and annotation overview. b Assembled transcripts size distribution. c Distribution of the top 10 species with most homologues to M. mercenaria. Transcripts were searched using BLASTx against NCBI nr database with a cutoff value of $\mathrm{E}<10 \mathrm{E}-03$ 
gigantea $(3,539)$ and the California sea slug Aplysia californica (1,931) (Fig. 1c). KEGG Orthology (KO) terms were assigned to 6,425 sequences and reference pathways were mapped to the KEGG database based on the assigned $\mathrm{KO}$ terms (Fig. 1a, Additional file 1). A total of 29,815 sequences were identified to match to at least one conserved protein domain in the InterPro database (Fig. 1a, Additional file 1).

Gene ontology (GO) assignments were used to classify functions of the predicted clam proteins. Based on sequence similarity (E-value of 10E-03), 13,584 sequences were assigned to at least one GO annotation (Fig. 1a, Additional file 1). As summarized in Fig. 2, a total of $8,168,4,600$ and 4,231 sequences were respectively categorized into the three main categories: biological process, cellular component, and molecular function at the second functional annotation level. The most dominant terms presented in the three categories are the "cellular process", "metabolic process", "binding", "catalytic activity", "cell", and "organelle". Very few transcripts were clustered into "rhythmic process", "cell killing", "protein tag", "channel regulator activity", "nucleoid" or "virion". It is noticeable that a good fraction of transcripts were clustered into the immunerelated categories of response to stimulus (503), immune system process (43) and biological adhesion (38). Those

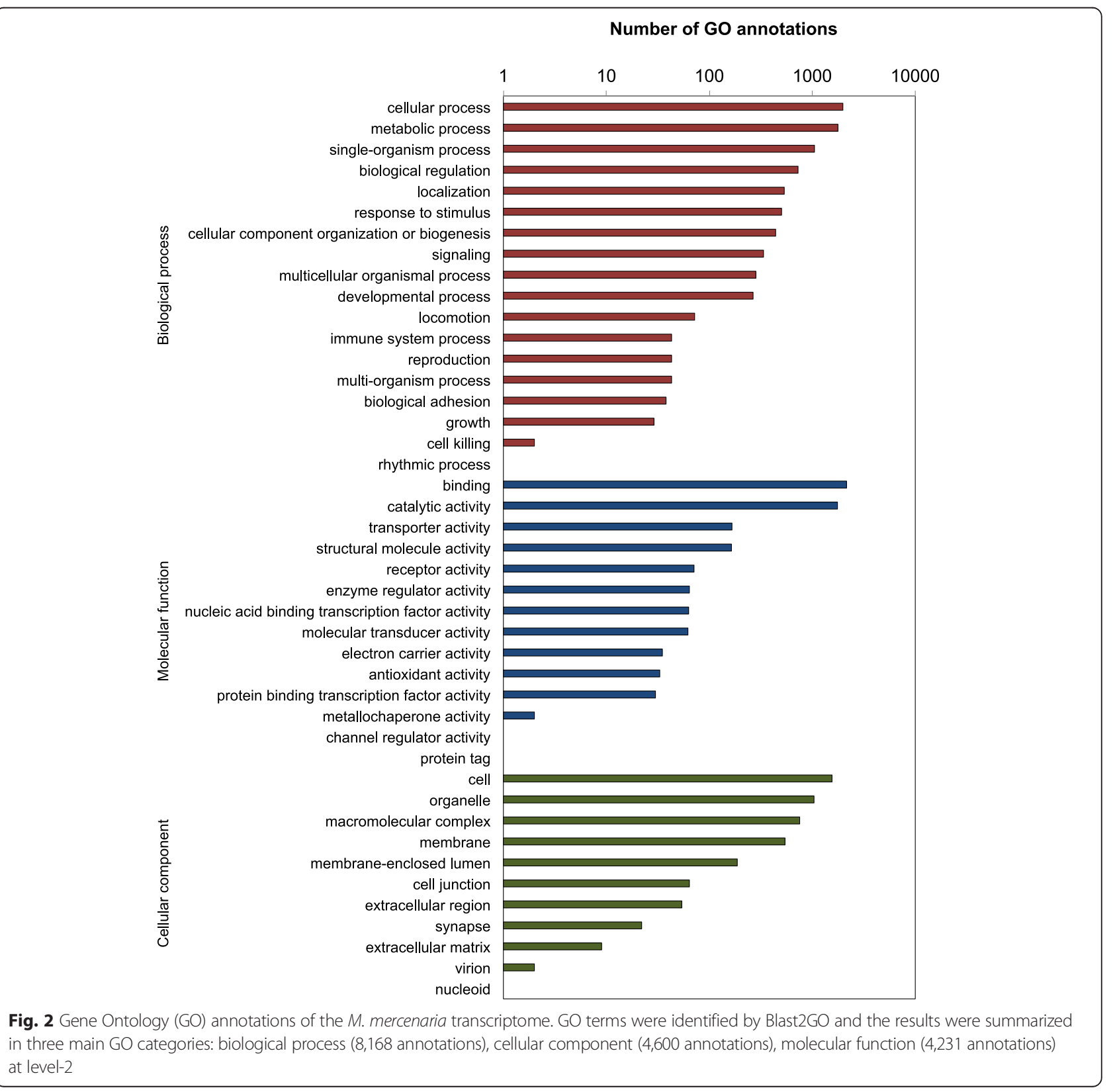


transcripts were of special interest given that they might be involved in the $M$. mercenaria defense and resistance toward QPX infection.

A significant portion $(69.7 \%)$ of M. mercenaria transcripts did not match any BlastX hit in NCBI nr database, in agreement with previous transcriptomic studies in mollusks [17-20]. Most of the unannotated transcripts may represent transcripts spanning untranslated mRNA regions, or transcripts containing only nonconserved protein domains $[21,22]$.

\section{Identification of differentially expressed transcripts}

The generated transcriptome was used as a reference for downstream investigations of global gene expression in the three different tissues of interest (nodule, nonnodule and healthy) to identify genes associated with $M$. mercenaria's focal and systemic immune response against QPX. A gene-isoform relationship was estimated using RSEM over Trinity output isoforms. Results showed that about $43 \%(27,021)$ of all the transcripts had 1 isoform, $19 \%(12,307)$ had 2 isoforms and $38 \%$ $(23,652)$ had 3 isoforms, suggesting extensive isoform diversity in $M$. mercenaria transcriptome. Transcript isoform variation could affect mRNA stability, localization and translation, as well as the production of protein variants that differ in localization or function [23].

By comparing the number of transcripts expressed in each sample, the contribution of specific samples to the analysis can be estimated. The highest number of expressed transcripts was found in the nodules of infected clams, which were closely followed by that found in the healthy clam samples (Fig. 3a). The lowest number of expressed transcripts came from non-nodule samples of QPX infected clams, with about 1,500 less transcripts expressed than the other two samples. Read coverage, which is critical in accurate determination of fold change, averaged 477, 422 and 509 reads per transcript for nodule, non-nodule and healthy tissue samples, respectively (Fig. 3b).

Statistical analysis by DEseq identified 3,131 differentially expressed (DE) transcripts from the pair-wise comparisons ( $\mid \log _{2}$ (fold change) $\mid>2$, adjusted $p$-value $<0.001$ ) between clam tissue samples (Fig. 4). In nodules, a total of 829 transcripts, including 408 over-expressed and 421 under-expressed transcripts were identified as compared to non-nodule samples of QPX infected clams. Compared to tissues from healthy clams, 1,591 DE transcripts were identified in nodules with 864 overexpressed and 727 under-expressed transcripts. Similarly, 1,681 DE transcripts were obtained from the comparison between healthy and non-nodule clam tissues, of which 513 and 1,168 were over- and under-expressed, respectively (Fig. 4, Additional file 3). A total of 1,694 of these DE transcripts had protein homologs found in NCBI $\mathrm{nr}$ database by Blastx searches (e-value <10E-03), which were further examined for their putative functions during

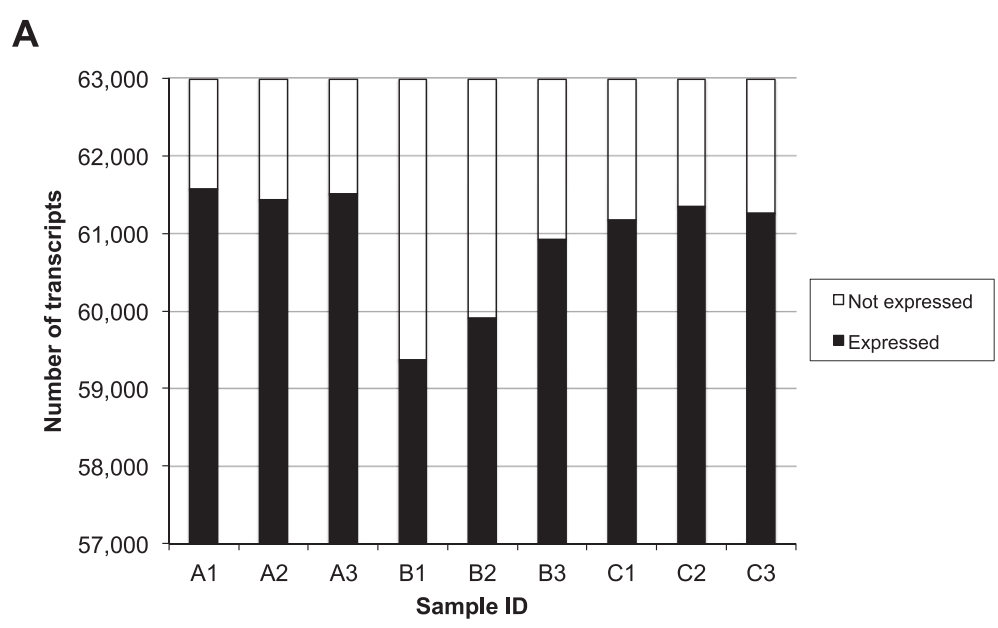

B

Transcripts expression and reads coverage of each sample library

\begin{tabular}{lllllllllll}
\hline & & \multicolumn{3}{c}{ Nodule } & \multicolumn{3}{c}{ Non-nodule } & \multicolumn{3}{c}{ Healthy } \\
\cline { 3 - 10 } & & A1 & A2 & A3 & B1 & B2 & B3 & C1 & C2 & C3 \\
\hline Reads coverage & Reads/transcript & 383 & 440 & 607 & 363 & 379 & 525 & 458 & 546 & 523 \\
& Average & 477 & & & 422 & & & 509 & & \\
& & & & & & & & & &
\end{tabular}

Fig. 3 Number of transcripts expressed and reads coverage in each sample. a The X-axis indicates the sample (A1-A3: nodule; B1-B3: non-nodule; C1-C3: healthy, refer to Table 1 for more details). The Y-axis indicates the number of transcripts expressed in the samples. $\mathbf{b}$ Summary statistics of the reads coverage in each sample 


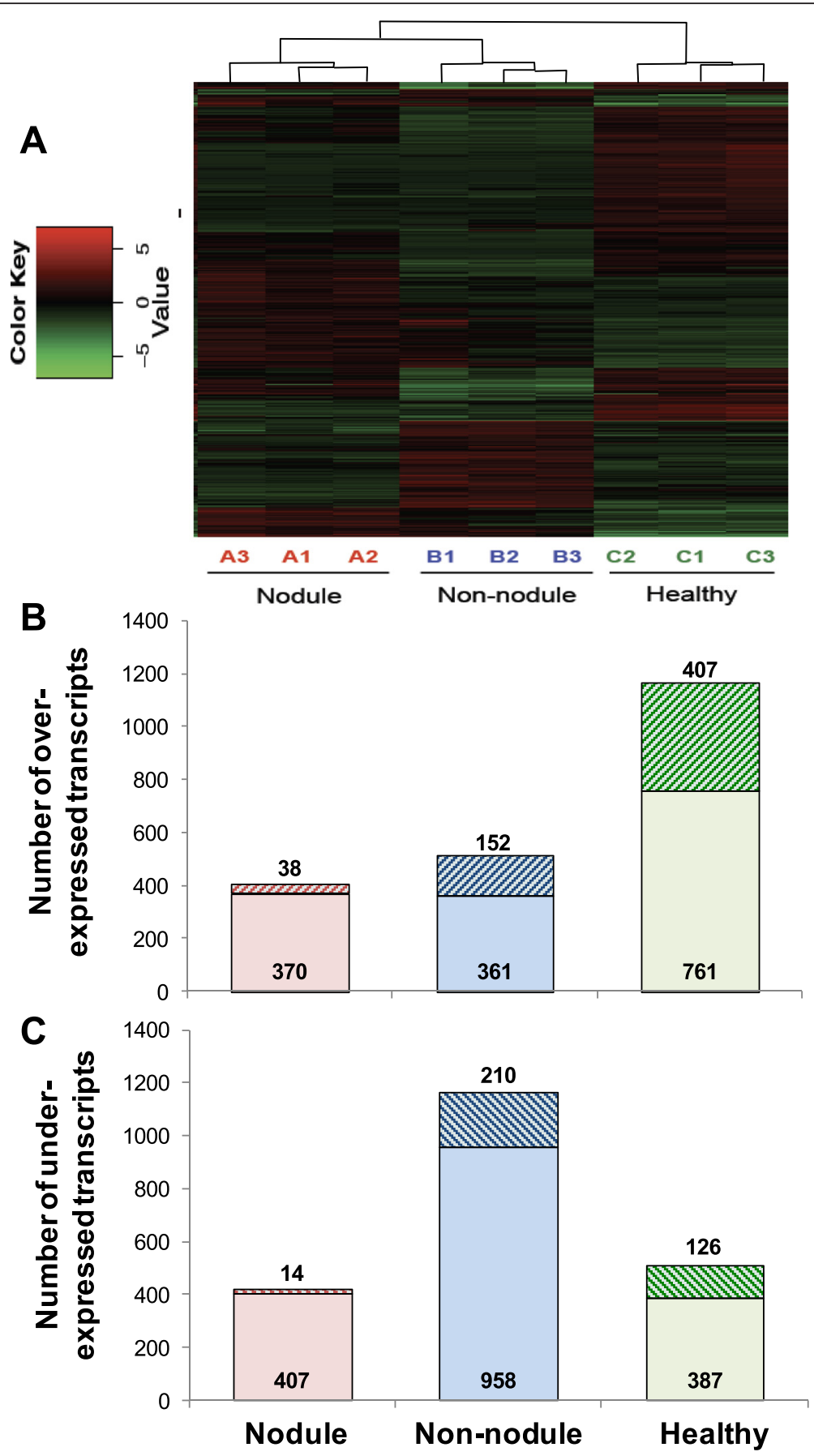

Fig. 4 Heatmap (a) and number of differentially expressed transcripts (b and $\mathbf{c}$ ) across all samples (FDR $\leq 0.001$, and $\mid \log _{2}$ (fold change) $>2$ ). Overexpressed transcripts are shown in red in A and are enumerated in B. Under-expressed transcripts are shown in green in A and are enumerated in C. Replicate biological samples are displayed in A (see Table 1 for more details). For B and C: the cross-shaded areas inside each bar represent the number of transcripts with higher (B) or lower (C) expression levels in a condition as compared to the other two (e.g., the expression levels of 38 focally over-expressed transcripts in B were higher in nodule than in non-nodule tissues and healthy clams)

M. mercenaria immune response toward QPX. Annotated transcripts were subsequently grouped into curated categories according to their biological functions based on the gene ontology (GO) terms and literature searches highlighting immune functions. DE transcript sets were further examined in reference to the assigned $\mathrm{KO}$ terms for the analysis of pathways regulation. Significantly enriched KEGG pathways were identified via the Fisher's exact test $(P<0.01)$ [24]. 
Here we specifically focus on DE transcripts drawn from comparisons between "nodule vs. non-nodule" and "non-nodule vs. healthy" tissue samples, which were respectively considered to reflect the transcriptomic changes caused by "focal" and "systemic" immune response of M. mercenaria toward QPX infection, respectively. The overview of DE transcripts drawn from these two responses is presented in Fig. 4 and Additional files 2 and 3.

\section{Differential expression of immune related transcripts Focal response}

Clam focal response reflected the alterations caused by direct clam-QPX interaction at the infection site (Additional file 2). QPX nodules are inflammatory masses resulting from massive hemocytes infiltration and encapsulation of parasite cells $[1,2]$. This process largely relies on the motility and adhesion properties of hemocytes, thus allowing these cells to migrate throughout the circulatory system and recruit to the infection site. Hemocytes can sense stimuli in host tissues through an array of cell surface receptors, and use these cues to adjust their behavior accordingly [25]. The activation of hemocytes requires the binding of specific ligands to the cell surface receptors, which subsequently initiate the transduction of extracellular signals into the cytoplasm via a variety of signaling pathways, thus inducing a series of hemocytemediated immune response such as phagocytosis, encapsulation (prominent response against QPX in clams), ROS production, as well as secretion of immune effectors and cytokines [26-28]. A collection of DE transcripts involved in these defense processes was identified during focal response, suggesting that strong and comprehensive hostpathogen interactions were taking places inside the QPX lesions (Table 2, Additional file 2). A large fraction of the DE transcripts of focal response was annotated as receptors or molecules with receptor activities, which putatively contribute to the host defense against QPX as (1) cell surface receptors expressed on hemocytes that mediate the recognition and phagocytosis or encapsulation of foreign entities through microbe associated molecular patterns (MAMP); (2) signaling receptors activating intracellular signaling cascades or (3) the soluble bridging molecules mediating the linkage between MAMPs and hemocytes [29]. Among those receptors, most are identified as pathogen pattern recognition receptors (PRRs), which include the C-type lectins (CTLs), the scavenger receptors (SRs) and the toll-like receptors (TLRs).

The C-type mannose receptor-2 (MRC2) identified during the focal response (Table 2) is a member of the C-type lectins (CTLs) superfamily, a large group of $\mathrm{Ca}^{2+}$-dependent carbohydrate-binding proteins that play crucial roles in innate immunity. CTLs recognize pathogens and facilitate their phagocytosis [30, 31] or encapsulation [32-34]. MRCs are also key regulators of inflammatory responses and contribute to the removal of harmful inflammatory agents [35, 36]. The 16-fold over-expression of MRC2 during the focal response suggested that active hemocyte encapsulation and local inflammation was induced by QPX at the infection lesions, which is consistent with the results of histopathological observations [1]. Another over-expressed CTL member, the perlucin-like protein, has been previously shown to trigger immune response in Manila clams during microbial infection [37].

Scavenger receptors (SRs) were also among the strongly over-expressed transcripts in infection foci (Table 2). These included somatomedin-b and thrombospondin type-1 domain-containing (RPE spondin), insulin-related peptide receptor, hemicentin-1, lysyl oxidase-like protein 2 and mam domain-containing glycosylphosphatidylinositol anchor protein 1 . SRs are structurally diverse PRRs that share the common function of recognizing oxidized or acetylated low-density lipoprotein (LDL) [38]. They contribute to innate immunity by recognizing MAMPs and mediating non-opsonic phagocytosis [29, 39]. They are extensively found on immune cells and are able to interact with both modified-host components and exogenous ligands, which makes SRs a key component in host defense, apoptosis, inflammation and lipoprotein homeostasis [40-42]. For example, scallop SRs bind not only to acetylated LDL but also to MAMP including lipopolysaccharides (LPS), peptidoglycans (PGN), mannan and zymosan particles [39]. The sea urchin genome encodes approximately 150 genes consisting of one or more scavenger receptor cysteine-rich (SRCR) domains [43], and the members of this gene family exhibit dynamic shifts in transcription after immune challenge [40, 44].

Our results also show an over-expression of TLR-1 and Toll-8/tollo in nodules (Table 2), which is in agreement with previous investigations showing up-regulation of TLRs in M. mercenaria mantle following QPX challenge [15]. TLRs are among the most ancient and conserved PRRs. They are expressed by immune cells and interact with a large variety of MAMPs. Bivalve TLRs have been characterized in the oyster C. gigas and the scallop C. farreri where they exhibited significant response to LPS stimulation [45, 46]. Transcriptional modulation of TLRs has also been reported in Ruditapes philippinarum and Mya arenaria following MAMPs stimulation and bacterial challenge $[47,48]$. Interestingly, Toll-8 (Tollo) has been shown to participate in Drosophila epithelial immunity where it mediates host cells communication that subsequently activates systemic immune responses [49]. This suggests that the Toll pathway could be one of the crucial pivoting links that allow coordination between focal and systemic immune components during infection.

The QPX nodules are formed as the result of granulomatous inflammation, which is a chronic inflammatory 
Table 2 Transcripts with annotated functions (GO terms) related to immune recognition, signaling and regulation that were differentially expressed during M. mercenaria focal response against QPX. Additional information on these transcripts is given in Additional file 2. "Inf" designates an infinite fold change calculated for focal response as the expression of that transcript in non-nodule tissue was equal to 0

\begin{tabular}{|c|c|c|c|c|}
\hline Transcripts ID & Annotation & Regul-ation & Fold change & Function/GOs \\
\hline \multicolumn{5}{|c|}{ Pathogen recognition receptors (PRRS) } \\
\hline \multicolumn{5}{|l|}{ Scavenger receptors } \\
\hline comp186077_c0_seq3 & $\begin{array}{l}\text { somatomedin-b and thrombospondin } \\
\text { type-1 domain-containing (RPE spondin) }\end{array}$ & Up & 71.3 & $\begin{array}{l}\text { F:scavenger receptor activity; P:immune } \\
\text { response; F:polysaccharide binding }\end{array}$ \\
\hline comp179365_c0_seq8 & insulin-related peptide receptor & Up & 11.5 & $\begin{array}{l}\text { F:scavenger receptor activity; P:transmembrane } \\
\text { receptor protein tyrosine kinase signaling pathway }\end{array}$ \\
\hline comp169098_c0_seq1 & hemicentin-1 & Up & 10.6 & F:scavenger receptor activity; P:cell adhesion \\
\hline comp169486_c0_seq1 & |ysyl oxidase-like protein 2 & Up & 22.5 & F:scavenger receptor activity; F:copper ion binding \\
\hline comp192413_c0_seq18 & $\begin{array}{l}\text { mam domain-containing } \\
\text { glycosylphosphatidylinositol } \\
\text { anchor protein } 1\end{array}$ & Up & $\operatorname{lnf}$ & $\begin{array}{l}\text { F:polysaccharide binding; F:scavenger } \\
\text { receptor activity; P:immune response }\end{array}$ \\
\hline \multicolumn{5}{|l|}{ C-type lectin (CTL) } \\
\hline comp176879_c0_seq8 & c-type mannose receptor 2 & Up & 15.8 & F:binding; F:carbohydrate binding \\
\hline comp190222_c0_seq3 & perlucin-like protein & Up & 49.1 & F:binding; F:carbohydrate binding; \\
\hline \multicolumn{5}{|l|}{ Toll-like receptors (TLRs) } \\
\hline comp189381_c0_seq1 & toll-like receptor 1 & Up & 16.5 & F:protein binding \\
\hline comp190056_c0_seq8 & toll-like receptor e precursor & Down & $\operatorname{lnf}$ & P:signal transduction; F:protein binding \\
\hline comp188195_c0_seq7 & toll-like receptor g precursor & Up & $\operatorname{lnf}$ & F:protein binding; P:signal transduction \\
\hline comp189381_c1_seq2 & cell surface receptor tollo (toll 8) & Up & 10.9 & P:signal transduction; F:protein binding \\
\hline \multicolumn{5}{|l|}{ Integrin } \\
\hline comp189082_c0_seq13 & integrin alpha 4 & Up & $\operatorname{lnf}$ & P:cell-cell adhesion; C:integrin complex \\
\hline comp189082_c0_seq24 & integrin alpha 4 & Up & 16.2 & P:cell-cell adhesion; C:integrin complex \\
\hline comp185789_c0_seq4 & integrin alpha-ps & Up & 13.7 & P:cell adhesion; C:integrin complex \\
\hline comp184975_c0_seq10 & integrin beta-1-like & Up & 12.4 & F:protein binding; F:receptor activity; \\
\hline \multicolumn{5}{|c|}{ Low-density lipoprotein (LDL) } \\
\hline comp181432_c3_seq2 & LDL receptor-related protein 12 & Up & 12.1 & - \\
\hline comp187193_c1_seq4 & LDL receptor-related protein 12 & Up & $\operatorname{lnf}$ & F:protein binding \\
\hline \multicolumn{5}{|c|}{ G-protein coupled receptor } \\
\hline comp177186_c1_seq2 & G-protein coupled receptor partial & Up & 13.0 & $\begin{array}{l}\text { P:cell surface receptor signaling pathway; } \\
\text { F:transmembrane signaling receptor activity }\end{array}$ \\
\hline comp191316_c2_seq1 & G-protein coupled receptor 64-like & Up & 28.1 & - \\
\hline comp177657_c2_seq5 & $\begin{array}{l}\text { guanine nucleotide-binding } \\
\text { protein subunit }\end{array}$ & Up & $\operatorname{lnf}$ & $\begin{array}{l}\text { P:G-protein coupled receptor signaling } \\
\text { pathway; F:signal transducer activity }\end{array}$ \\
\hline comp187628_c0_seq44 & substance-k (neurokinin) receptor & Up & 9.1 & $\begin{array}{l}\text { P:G-protein coupled receptor signaling pathway; } \\
\text { F:G-protein coupled receptor activity; }\end{array}$ \\
\hline comp192446_c0_seq4 & orexin receptor type 2 & Up & 12.4 & P:G-protein coupled receptor signaling pathway; \\
\hline \multicolumn{5}{|l|}{$\begin{array}{l}\text { Immune signaling and } \\
\text { cell communication }\end{array}$} \\
\hline \multicolumn{5}{|l|}{ Phosphatase and kinases } \\
\hline comp192546_c5_seq4 & immunoglobulin i-set domain protein & Up & 13.2 & F:protein serine/threonine kinase activity \\
\hline comp165267_c0_seq3 & $\begin{array}{l}\text { von willebrand factor type egf and } \\
\text { pentraxin domain-containing protein }\end{array}$ & Up & 11.0 & F:protein tyrosine kinase activity \\
\hline comp191437_c1_seq4 & focal adhesion kinase 1 & Up & 19.7 & F:signal transducer activity; \\
\hline comp188649_c0_seq3 & $\begin{array}{l}\text { calcium calmodulin-dependent } \\
\text { protein kinase }\end{array}$ & Up & 9.3 & F:calmodulin-dependent protein kinase activity \\
\hline comp190317_c0_seq7 & neuronal cell adhesion & Down & -13.3 & F:rhodopsin kinase activity \\
\hline
\end{tabular}


Table 2 Transcripts with annotated functions ( $G O$ terms) related to immune recognition, signaling and regulation that were differentially expressed during M. mercenaria focal response against QPX. Additional information on these transcripts is given in Additional file 2. "Inf" designates an infinite fold change calculated for focal response as the expression of that transcript in non-nodule tissue was equal to 0 (Continued)

\begin{tabular}{|c|c|c|c|c|}
\hline \multicolumn{5}{|l|}{ Rho signaling } \\
\hline comp189234_c1_seq21 & $\begin{array}{l}\text { rho gtpase-activating protein } \\
15 \text {-like isoform } \times 3\end{array}$ & Up & 18.1 & P:signal transduction \\
\hline comp185122_c1_seq7 & rho gtpase-activating protein 24 & Up & 27.1 & P:signal transduction \\
\hline comp187736_c1_seq25 & rho-related protein raca & Up & Inf & F:protein binding \\
\hline comp188711_c0_seq2 & rho-associated protein kinase 2 & Up & Inf & P:intracellular signal transduction \\
\hline \multicolumn{5}{|l|}{ Ubiquitin pathway } \\
\hline comp190451_c2_seq9 & e3 ubiquitin-protein ligase hectd1 & Up & 164.9 & $\begin{array}{l}\text { F:ubiquitin-protein ligase activity; } \\
\text { F:metal ion binding }\end{array}$ \\
\hline comp184512_c0_seq2 & e3 ubiquitin-protein ligase march6 & Up & 15.0 & F:zinc ion binding \\
\hline comp192081_c0_seq1 & e3 ubiquitin-protein ligase ubr3 & Up & 8.7 & - \\
\hline comp179031_c0_seq4 & cop9 signalosome complex subunit 5 & Up & $\operatorname{lnf}$ & F:protein binding \\
\hline comp179031_c0_seq2 & cop9 signalosome complex subunit 5 & Up & $\operatorname{lnf}$ & F:protein binding \\
\hline \multicolumn{5}{|l|}{ Wnt and Notch pathway } \\
\hline comp185090_c0_seq10 & tyrosine-protein kinase ryk & Up & 26.3 & P:Wnt receptor signaling pathway \\
\hline comp187449_c0_seq2 & fizzy-like protein & Up & 13.0 & F:protein binding \\
\hline comp175460_c0_seq1 & neurogenic locus notch & Up & 11.7 & P:Notch signaling pathway; \\
\hline comp182793_c0_seq5 & neurogenic locus notch protein & Up & 9.6 & P:G-protein coupled receptor signaling pathway \\
\hline comp192565_c0_seq10 & nicastrin-like protein & Up & 12.6 & P:protein processing \\
\hline \multicolumn{5}{|c|}{ Calcium mediated signal transduction } \\
\hline comp183265_c0_seq1 & calmodulin 3b (phosphorylase delta) & Down & -11.6 & F:calcium ion binding \\
\hline comp191993_c0_seq4 & $\begin{array}{l}\text { EF-hand Ca-binding domain-containing } \\
\text { protein } 5\end{array}$ & Down & -10.9 & - \\
\hline comp191855_c0_seq2 & $\begin{array}{l}\text { EF-hand Ca-binding domain-containing } \\
\text { protein } 6\end{array}$ & Down & -15.9 & - \\
\hline \multicolumn{5}{|l|}{ Complement pathway } \\
\hline comp165285_c0_seq7 & macrophage-expressed gene 1 & Up & 11.5 & - \\
\hline \multicolumn{5}{|l|}{ Signal transducer } \\
\hline comp182953_c0_seq4 & $\begin{array}{l}\text { signal recognition particle } \\
\text { receptor subunit alpha }\end{array}$ & Up & 13.8 & F:signal recognition particle binding \\
\hline comp182953_c0_seq5 & $\begin{array}{l}\text { signal recognition particle } \\
\text { receptor subunit alpha }\end{array}$ & Up & 73.0 & F:signal recognition particle binding \\
\hline comp171563_c0_seq4 & gtp-binding nuclear protein & Up & $\operatorname{lnf}$ & P:small GTPase mediated signal transduction \\
\hline comp188686_c0_seq19 & neuralized pats1 & Down & -758.9 & P:small GTPase mediated signal transduction \\
\hline comp189853_c0_seq1 & unc $5 \mathrm{c}$-like protein & Down & -12.5 & P:signal transduction \\
\hline \multicolumn{5}{|l|}{ Cell death regulation } \\
\hline \multicolumn{5}{|l|}{ Apoptosis process } \\
\hline comp175357_c1_seq16 & $\begin{array}{l}\text { solute carrier family } 25 \\
\text { member } 38 \text {-like isoform } 1\end{array}$ & Down & -25.0 & P:transmembrane transport \\
\hline comp191590_c0_seq3 & $\begin{array}{l}\text { p53-induced protein with } \\
\text { a death domain isoform }\end{array}$ & Down & -39.1 & F:protein binding; P:signal transduction \\
\hline comp191147_c0_seq62 & inhibitor of apoptosis & Up & 24.6 & - \\
\hline comp191147_c0_seq70 & inhibitor of apoptosis & Up & $\operatorname{lnf}$ & F:metal ion binding; F:zinc ion binding; \\
\hline comp191055_c2_seq4 & programmed cell death protein 10 & Up & 367.1 & - \\
\hline comp190690_c2_seq9 & cell death abnormality protein 1-like & Up & $\operatorname{lnf}$ & F:binding; F:zinc ion binding \\
\hline
\end{tabular}


Table 2 Transcripts with annotated functions (GO terms) related to immune recognition, signaling and regulation that were differentially expressed during M. mercenaria focal response against QPX. Additional information on these transcripts is given in Additional file 2. "Inf" designates an infinite fold change calculated for focal response as the expression of that transcript in non-nodule tissue was equal to 0 (Continued)

\begin{tabular}{|c|c|c|c|c|}
\hline comp186101_c3_seq4 & $\begin{array}{l}\text { 3-hydroxy-3-methylglutaryl- } \\
\text { coenzyme A reductase }\end{array}$ & Down & -10.8 & $\begin{array}{l}\text { P:positive regulation of apoptotic process; P:negative } \\
\text { regulation of wound healing; P:oxidation- } \\
\text { reduction process; }\end{array}$ \\
\hline \multicolumn{5}{|c|}{ Tumor necrosis factor (TNF) } \\
\hline comp182922_c0_seq5 & TNF ligand superfamily member 10-like & Up & 8.5 & - \\
\hline \multirow[t]{2}{*}{ comp176786_c2_seq3 } & TNF-like protein & Down & -26.3 & $\begin{array}{l}\text { F:tumor necrosis factor receptor } \\
\text { binding; P:immune response }\end{array}$ \\
\hline & TNF ligand superfamily member 10 -like & Up & 8.5 & - \\
\hline
\end{tabular}

reaction characterized by focal accumulation of activated immune cells to isolate the invading agent $[50,51]$. The formation of granuloma requires local recruitment of hemocytes at the site of infection to execute extracellular defense processes around the invaders [50]. An array of transcripts associated with cell migration, adhesion and proliferation was regulated in nodules, including Gprotein coupled receptors (GPCRs) and integrins families (Table 2). GPCRs regulate inflammatory response via binding to chemokines and chemoattractants, thus activating pathways mediating hemocyte migration and adhesion [52]. They also activate transcription factors in immune cells, thus modulating the synthesis and secretion of certain pro- or anti-inflammatory substances [53]. On the other hand, integrins represent a major group of cell adhesion mediators [54]. They not only modulate the cell-cell and cell-extracellular matrix adhesion, but also affect multiple signal transduction cascades regulating cell survival and proliferation [54]. Overexpression of GPCRs and integrins in nodules suggests their role in hemocytes adhesion and aggregation associated with the formation of granuloma [50, 55].

Several enzymes regulating ROS production were also over-expressed during focal response (Table 3). These included a dual oxidase, which is a key component mediating host-microbe interactions in mucosa [56, 57]. Dual oxidase regulates oxidative burst and ROS production in the gill muscosa of the shrimp Marsupenaeus japonicus, favoring shrimp survivorship during viral infections [58]. Interestingly, transcripts of dual oxidase were only expressed in nodules, suggesting this enzyme was induced upon direct clam-QPX interactions as a part of the mantle mucosa-related immune response. Other transcripts associated with oxidation-reduction processes also exhibited somewhat nodule-exclusive pattern, including the allene oxide synthase-lipoxygenase (AOSL), lysyl oxidase-like protein (LOXL), ww domaincontaining oxidoreductase (WWOX), c-terminal binding protein (CtBP), isocitrate dehydrogenase (ICD) and methylenetetrahydrofolate reductase (MTHFR). These molecules are important for maintaining the redox homeostasis of extracellular environment as they are key regulators for oxi-reduction reactions. Over-expression of these transcripts in nodules suggests the need for the host to timely balance out excessive ROS and other toxic intermediates produced during interaction with QPX. In addition to redox-regulation, many of these molecules also take part in the immune modulation indirectly. For example, AOSL play a role in coral immunity by controlling the production of the inflammation regulator arachidonic acid during apoptosis [59], and LOXL acts both as a scavenger receptor and regulator for extracellular matrix remodeling that initiate hemocyte migration and tissue regeneration [60], while WWOX was shown to promote proliferation of immune cells through inhibition of their apoptosis $[61,62]$. In addition, ICD, MTHFR and cytochrome p450 are major detoxification enzymes $[63,64]$. In fact, immune cells and their secreted effectors require the proper redox state in the extracellular environments to exert their immune functions, which makes the maintenance of redox homeostasis essential for persistent and effective host defense [65, 66]. This is particularly true in the case of QPX disease where the neutralization of parasites depends on extracellular killing pathways [26].

Apoptosis is an essential host mechanism to effectively remove damaged and infected cells without causing inflammatory destructions to surrounding tissues $[67,68]$. Interestingly, apoptosis seems to be largely inhibited during $M$. mercenaria focal response, as shown by the under-expression of pro-apoptosis transcripts (Table 2). For example, the tumor necrosis factor (TNF)-like protein and 3-hydroxy-3-methylglutaryl-coenzyme A (HMG-CoA) reductase-like protein were significantly under-expressed in nodules. Similarly, the pro-apoptotic p53-induced protein and solute carrier family 25 member protein were also under-expressed in nodules. Meanwhile, inhibitor of apoptosis protein (IAP) was overexpressed in nodules. IAPs regulate immune cell expansion and survival in highly inflammatory environments in mammals [69] and they may share similar function in clams by preventing hemocytes from death during 
Table 3 Transcripts with putative functions (GO terms) related to immune effectors that were differentially expressed during $M$. mercenaria focal response against QPX. Additional information on these transcripts is given in Additional file 2. "Inf" designates an infinite fold change calculated for focal response as the expression of that transcript in non-nodule tissue was equal to 0

\begin{tabular}{|c|c|c|c|c|}
\hline Transcripts ID & Annotation & $\begin{array}{l}\text { Regula- } \\
\text { tion }\end{array}$ & $\begin{array}{l}\text { Fold } \\
\text { change }\end{array}$ & Function/GOs \\
\hline \multicolumn{5}{|c|}{ Oxidation-reduction processes } \\
\hline comp186178_c0_seq12 & dual oxidase & Up & Inf & $\begin{array}{l}\text { P:response to oxidative stress; P:oxidation- } \\
\text { reduction process; F:peroxidase activity }\end{array}$ \\
\hline comp185478_c0_seq4 & $\begin{array}{l}\text { allene oxide synthase- } \\
\text { lipoxygenase protein }\end{array}$ & Up & 10.0 & $\begin{array}{l}\text { P:oxidation-reduction process; F:oxidoreductase } \\
\text { activity; F:metal ion binding; }\end{array}$ \\
\hline comp186926_c0_seq7 & c-terminal-binding protein & Up & Inf & P:oxidation-reduction process; F:NAD binding; \\
\hline comp187462_c1_seq6 & chorion peroxidase & Up & 11.8 & $\begin{array}{l}\text { P:oxidation-reduction process; P:response } \\
\text { to oxidative stress; F:peroxidase activity }\end{array}$ \\
\hline comp157634_c0_seq8 & cytochrome p450 & Up & 178.6 & $\begin{array}{l}\text { P:oxidation-reduction process; F:oxidoreductase } \\
\text { activity; F:iron ion binding }\end{array}$ \\
\hline comp183426_c1_seq2 & $\begin{array}{l}\text { dbh-like monooxygenase } \\
\text { protein 1-like protein }\end{array}$ & Up & 24.8 & $\begin{array}{l}\text { P:oxidation-reduction process; F:dopamine } \\
\text { beta-monooxygenase activity; } \\
\text { F:oxidoreductase activity }\end{array}$ \\
\hline comp188723_c0_seq1 & isocitrate dehydrogenase & Up & Inf & $\begin{array}{l}\text { P:oxidation-reduction process; F:magnesium } \\
\text { ion binding; F:NAD binding }\end{array}$ \\
\hline comp169486_c0_seq1 & lysyl oxidase-like protein 2 & Up & 22.5 & $\begin{array}{l}\text { F:oxidoreductase activity; P:oxidation-reduction } \\
\text { process; F:scavenger receptor activity }\end{array}$ \\
\hline comp192316_c0_seq4 & $\begin{array}{l}\text { methylenetetrahydrofolate } \\
\text { reductase }\end{array}$ & Up & Inf & $\begin{array}{l}\text { P:oxidation-reduction process; F:methylenetetrahydrofolate } \\
\text { reductase (NADPH) activity; }\end{array}$ \\
\hline comp189621_c0_seq4 & $\begin{array}{l}\text { procollagen-oxoglutarate } \\
\text { 5-dioxygenase } 3\end{array}$ & Up & 9.7 & P:oxidation-reduction process; F:iron ion binding \\
\hline comp185148_c0_seq6 & $\begin{array}{l}\text { ww domain-containing } \\
\text { oxidoreductase }\end{array}$ & Up & Inf & F:oxidoreductase activity; P:metabolic process \\
\hline \multicolumn{5}{|l|}{ Protease } \\
\hline comp184786_c1_seq4 & fur protein precursor & Up & Inf & F:serine-type endopeptidase activity \\
\hline comp180950_c0_seq5 & $\begin{array}{l}\text { lysosomal protective } \\
\text { protein precursor }\end{array}$ & Up & 47.1 & F:serine-type carboxypeptidase activity \\
\hline comp180950_c0_seq2 & $\begin{array}{l}\text { lysosomal protective } \\
\text { protein precursor }\end{array}$ & Up & 26.9 & F:serine-type carboxypeptidase activity \\
\hline comp189961_c0_seq12 & $\begin{array}{l}\mathrm{N} \text {-acetylated-alpha-linked } \\
\text { acidic dipeptidase } 2\end{array}$ & Up & 16.3 & F:metallopeptidase activity; \\
\hline comp178551_c1_seq1 & $\begin{array}{l}\text { membrane metallo- } \\
\text { endopeptidase-like 1-like }\end{array}$ & Up & Inf & F:metalloendopeptidase activity; \\
\hline comp184011_c0_seq4 & blastula protease 10 & Up & 8.1 & F:metalloendopeptidase activity; \\
\hline comp191868_c1_seq1 & matrix metalloproteinase-19 & Up & 59.1 & - \\
\hline comp174947_c0_seq3 & $\begin{array}{l}\text { isoaspartyl peptidase } \\
\text { l-asparaginase-like }\end{array}$ & Up & Inf & F:hydrolase activity \\
\hline comp183848_c0_seq3 & kyphoscoliosis peptidase & Down & -8.7 & P:microtubule-based movement \\
\hline comp188831_c0_seq3 & $\begin{array}{l}\text { puromycin-sensitive } \\
\text { aminopeptidase-like } \\
\text { isoform }\end{array}$ & Down & -65.5 & F:metallopeptidase activity; \\
\hline comp191458_c3_seq5 & $\begin{array}{l}\text { aspartic protease with reverse } \\
\text { transcriptase activity }\end{array}$ & Down & -245.4 & F:aspartic-type endopeptidase activity; \\
\hline \multicolumn{5}{|l|}{ Protease inhibitor } \\
\hline comp189919_c1_seq4 & alpha macroglobulin & Up & 21.9 & F:endopeptidase inhibitor activity \\
\hline comp189919_c1_seq2 & alpha macroglobulin & Up & 12.4 & F:endopeptidase inhibitor activity \\
\hline comp181286_c4_seq1 & thioester-containing protein & Up & 10.2 & F:endopeptidase inhibitor activity \\
\hline comp191416_c1_seq2 & thioester-containing protein-a & Up & 14.8 & F:endopeptidase inhibitor activity \\
\hline comp191416_c1_seq1 & thioester-containing protein- $b$ & Up & 9.5 & F:endopeptidase inhibitor activity \\
\hline
\end{tabular}


Table 3 Transcripts with putative functions (GO terms) related to immune effectors that were differentially expressed during $M$. mercenaria focal response against QPX. Additional information on these transcripts is given in Additional file 2. "Inf" designates an infinite fold change calculated for focal response as the expression of that transcript in non-nodule tissue was equal to 0 (Continued)

\begin{tabular}{|c|c|c|c|c|}
\hline comp192366_c0_seq1 & thioester-containing protein-c & Up & 32.5 & F:endopeptidase inhibitor activity \\
\hline comp191416_c0_seq5 & thioester-containing protein-e & Up & 12.9 & F:endopeptidase inhibitor activity \\
\hline comp191416_c0_seq4 & thioester-containing protein-e & Up & 22.4 & F:endopeptidase inhibitor activity \\
\hline \multicolumn{5}{|c|}{ Ion transporter and sequester } \\
\hline comp190604_c1_seq1 & ceruloplasmin precursor & Up & 21.8 & $\begin{array}{l}\text { P:copper ion transport; P:cellular iron ion homeostasis; } \\
\text { F:ferroxidase activity }\end{array}$ \\
\hline comp182612_c1_seq1 & ferric-chelate reductase 1 & Up & 16.7 & Iron transfer \\
\hline comp180332_c1_seq1 & ferric-chelate reductase 1 -like & Up & 11.8 & Iron transfer \\
\hline comp177359_c0_seq1 & selenium binding protein & Down & -11.0 & P:protein transport; F:selenium binding \\
\hline comp174164_c0_seq20 & divalent metal transporter & Up & 328.2 & P:transport; F:transporter activity \\
\hline \multicolumn{5}{|l|}{ Wound repair } \\
\hline comp185425_c1_seq1 & $\begin{array}{l}\text { actin-related protein } 23 \\
\text { complex subunit } 5 \text {-like }\end{array}$ & Up & Inf & C:cytoskeleton; P:regulation of actin filament polymerization \\
\hline comp93954_c0_seq1 & $\begin{array}{l}\text { extracellular matrix protein } 2 \\
\text { isoform } 1\end{array}$ & Up & 25.9 & F:protein binding \\
\hline comp188753_c5_seq1 & cartilage matrix protein & Up & 25.8 & $\begin{array}{l}\text { F:chitin binding; P:chitin metabolic } \\
\text { process; C:extracellular region }\end{array}$ \\
\hline comp142858_c0_seq1 & $\begin{array}{l}\text { epidermal growth factor-like } \\
\text { protein 8-like }\end{array}$ & Up & 18.5 & F:protein binding; F:calcium ion binding \\
\hline comp192650_c1_seq4 & $\begin{array}{l}\text { multiple epidermal growth } \\
\text { factor-like domains } 6\end{array}$ & Up & 21.9 & F:protein binding \\
\hline comp186665_c0_seq1 & thrombospondin- partial & Up & 106.9 & F:protein binding \\
\hline comp186665_c0_seq4 & thrombospondin- partial & Up & 38.0 & F:protein binding \\
\hline comp184960_c0_seq4 & septin-7-like isoform 8 & Up & Inf & P:cell cycle; F:GTP binding; C:septin complex \\
\hline comp184960_c0_seq42 & septin-7-like isoform 6 & Up & 8.9 & - \\
\hline
\end{tabular}

interaction with QPX. In fact, ROS production during parasite killing may trigger apoptotic cell death in molluscs [67], and proper control of apoptosis mechanisms is required to maintain cellular homeostasis during immune response. This suspected inhibition of host apoptosis is supported by the above-mentioned overexpression of integrins, as these were shown to protect cells from apoptosis and induce anti-apoptotic pathways during cell adhesion and spreading in the snails Lymnaea stagnalis [70] and B. glabrata [71].

Infection and tissue injury trigger host immune responses via immune signaling pathways [72], by activating transcription factors and initiating the production of immune effectors and regulators. Immune signaling pathways identified in mollusks include Toll, MAPK/ JNK and JAK/STAT signaling pathways [27, 47, 73, 74]. During $M$. mercenaria focal response to QPX, a variety of transcripts encoding kinases and phosphatases were over-expressed (Table 2), suggesting the involvement of MAPK and other kinase-mediated cascades in regulating the focal inflammatory response [75], whereas the under-expression of EF-hand domain containing protein and calmodulin may indicate the suppression of calcium-regulated pathways [76]. Overexpression of E3 ubiquitin-protein ligase and its upstream regulator COP9 signalosome suggests the activation of the damage surveillance ubiquitin/proteasome pathway [77-79]. In parallel, the over-expression of rho GTPase and rho kinase suggests the induction of anti-apoptotic Rhomediated signaling pathway $[80,81]$ and reinforce the idea that apoptotic inhibition is extensively initiated by M. mercenaria to help fight QPX. However, a very limited number of DE transcripts was detected in relation to those conventional signaling pathways of innate immunity, such as the complement pathway and the Toll/ TLR pathway. Only 1 transcript encoding macrophageexpressed gene protein 1 (MPEG1), a putative member of the complement pathway $[82,83]$, was differentially expressed in QPX nodules. As for the Toll/TLR pathway, only a few receptors were identified (Table 2) but none of their downstream components.

Interestingly, components of the Notch and the Wnt signaling pathways were over-expressed during focal response (Table 2). These included two putative Notch family members, the neurogenic locus Notch protein and the mediator protein nicastrin, and the tyrosin- 
protein kinase RYK which belongs to the Wnt pathway. Wnt signaling pathway regulates many cellular immune processes and is evolutionarily conserved across taxa $[84,85]$. Wnt signaling has been intensively exploited for its regulatory functions during wound healing and tissue regeneration [86, 87], so its over-expression may be related to wound healing to repair damage resulting from tissue digestion by the parasite or tissue necrosis. The Notch signaling network was scarcely explored in bivalves even though it has been reported to be associated with several aspects of immune response in mammals [88], especially in regulating granulomatous reactions to foreign bodies [89]. As an evolutionarily conserved pathway involved in modulating the intercellular signaling, the Notch pathway presumably shares an equally important role in $M$. mercenaria by modulating the formation of granuloma. Notch signaling triggers macrophage expression of genes involved in pro-inflammatory responses [90], but can suppress inflammation responses triggered by canonical TLR cascade [91], in agreement with our observations. These tightly regulated mechanisms ensure tailored immune responses against different pathogens and are crucial for the host to achieve high immune efficiency while avoiding excessive immune activation and self-inflicted damages.

Several proteases (A.K.A proteinases, peptidases) were also differentially regulated during $M$. mercenaria focal response against QPX. These proteases mostly belong to the serine and metallo protease families and were generally over-expressed in QPX nodules (Table 3). Proteases serve as key immune modulators partially through their ability to digest and remodel the extracellular matrix and tissues associated with hemocyte activation [92]. Commonly associated with lysosomes and granules of inflammatory cells, serine proteases participate in immune regulation either directly by degradation of pathogens or indirectly through activation of cell surface receptors and signal molecules [92-94]. The function of metalloproteases in immune regulation is even more diverse, acting as immune effectors, signal transducers, and mediators of immune cell development and migration [95]. Metalloproteases are also known to be involved in many pro-inflammatory pathways, particularly in the Notch pathway where they act as a type of downstream element to Notch [96].

At the same time, immune effectors with known universal protease inhibitor activities, such as alpha2macroglobulin $(\alpha 2 \mathrm{M})$ and thioester-containing protein (TEP, a subfamily of $\alpha 2 \mathrm{M}$ ), were also collectively overexpressed in QPX nodules (Table 3). The $\alpha 2 \mathrm{M}$ superfamily inhibits peptidases of diverse origins $[97,98]$. The simultaneous over-expression of proteases and protease inhibitors may reflect a finely adjusted defense response of $M$. mercenaria to maintain homeostasis and regulate self- and pathogen-derived proteases, as shown in other host-pathogen systems [92, 99], including bivalves [100]. Proteases have been identified as major virulence factors of QPX [101, 102], and are thought to degrade host proteinaceous immune effectors and hydrolyze host tissues to fulfill nutritional requirements. Therefore, inhibition of pathogen proteases contribute to host protection, and was shown to represent a determinant factor for resistance against infectious diseases in bivalves [103-106]. In parallel, TEPs have been extensively studied in mollusk immunity $[99,107]$, and beside their function as protease inhibitors, they also play a role as PRRs or opsonins to facilitate microbial phagocytosis and encapsulation. Consistent over-expression of TEPs was noted in this study in agreement with findings following experimental infection with QPX [15]. These results support a critical role of TEPs in clam immune response against QPX either via the protease-inhibitor activity of these proteins, or by mediating parasite encapsulation, or both.

In addition, several metal ion transporters were overexpressed in nodules (Table 3), including the putative copper ion binding protein ceruloplasmin precursor, the transferrin enzyme ferric-chelate reductase and the divalent metal transporter (A.K.A natural resistanceassociated macrophage protein; 328 fold increase). These molecules contribute to host defense by controlling the supply of essential micronutrients in the vicinity of infection sites thus reducing parasite survival $[108,109]$ and favoring the production of antimicrobial factors [110]. It is noteworthy to point out that some of the focally over-expressed transcripts might be partly driven by the dramatic increase of hemocyte proportion within tissues in the vicinity of infection foci as compared to the surrounding host tissues $[26,111]$. This would be especially the case for transcripts known to be highly expressed in hemocytes, such as the cell surface PRRs, secreted humoral immune effectors, cell signal transducers and enzymes associated with ROS production.

\section{Systemic response}

Significant transcriptomic regulations were observed during $M$. mercenaria systemic immune response against QPX, with a total of 1,681 DE transcripts, which is about two times the number of focal DE transcripts (829). However, only about one third of the DE transcripts (513) were over-expressed in response to QPX infection, the larger remaining part $(1,168)$ represented significantly under-expressed transcripts (Fig. 4, Additional file 3), possibly due to the chronic stress imposed by the infection. Transcriptome-wide depression has been demonstrated in many marine invertebrates as the result of pathogenic or environmental stress [18, 59, 112-114]. A considerable number of systemically underexpressed transcripts was related to metabolism and 
biomineralization, which could be the result of host resource allocation during on-going infection. The chronic inflammation induced by infection likely created extra energy demands, which require resources being allocated from other physiological processes, such as growth and reproduction, to immune processes which is critical for the survivorship of the host. In fact, slow growth and lower tissue conditions are frequently observed in QPXinfected $M$. mercenaria [2], and similar energy trade-offs existed between immune defense and other energy expenditure pathways [115-118]. Interestingly, a suite of transcripts over-expressed during focal response was significantly under-expressed in non-nodule tissues as compared to healthy clams (Additional file 3), which included several immune effectors and mediators associated with nodule formation and focal inflammation (e.g., integrins, notch proteins and peroxidases). In fact, maintaining high levels of these focally-induced molecules could be costly and dangerous as some are toxic to both the parasite and the host, so their production must be restrained within areas where they can directly exert defense function, and reduced outside the infection foci to minimize risks of undesirable effects on the host $[116,117]$.

On the other hand, systemically over-expressed transcripts included stress proteins and other soluble immune factors such as lysozyme (c-type lysozyme 2$)$, lectins ( $\mathrm{C} 1 \mathrm{q}$ domain containing protein, macrophage mannose receptor 1 , low affinity immunoglobulin epsilon fc receptor), AMP (hemocyte defensin), proteases (cathepsin K, calpain 11, isoaspartyl peptidase/L-asparaginase, ASRGL, counting factor associated protein d) and ferric-chelate reductase (Table 4, Additional file 3). Over-expression of host stress proteins, such as heat shock proteins (HSP 70, HSP 90) and universal stress protein (USP) was also noted, in agreement with observations made during infection in other bivalve species [119-121]. Increased levels of stress proteins provide host cells with protection against incorrect protein folding caused by infection, inflammation, oxidative stress and other destructive events $[122,123]$. The systemic over-expression of soluble immune effectors (e.g., humoral proteins) may help maintaining comparatively high immune capacity to prevent the spread of QPX (or secondary pathogens) throughout the host. In addition, transcripts of antiapoptotic factors (IL17, deoxyguanosine, baculoviral map repeat-containing proteins) were also overexpressed during the systemic response, indicating that anti-apoptotic processes noted during focal response are not limited to the infection foci.

Pathway alterations during M. mercenaria's response to QPX Transcriptomic alterations during both focal and systemic response discussed above were also highlighted in the pathway enrichment analysis of the DE transcripts.
This analysis aims at extracting an overview of phenotypic changes on the underlying functional level, to reduce the complexity of biological information given by the long lists of DE genes/transcripts [124]. The KEGG pathways of focal adhesion (04510), ECM-receptor interaction (04512), Notch signaling pathway (04330) and apoptosis (0421) were significantly over-represented during both focal and systemic response (Fig. 5), even though fold enrichment were generally higher during the focal response. Other immune-related pathways particularly enriched during the focal response included regulation of actin cytoskeleton (04810), cell adhesion molecules (CAMs, 04514), the leukocyte transendothelial migration (04670), complement and coagulation cascade (04610) and Wnt signaling pathway (04310). These pathways are critically involved in the immune cells activation during migration, attachment and parasite encapsulation, which serve as the underlying mechanisms for nodule formation and QPX killing. On the other hand, basic metabolic pathways such as the citrate cycle (TCA) and pyruvate metabolism were specially enriched during the systemic response. These alterations were largely in accordance with the under-expression of metabolism-associated DE transcripts in infected tissue compared to the healthy tissue (Fig. 4), possibly reflecting changes in the energy allocation strategy during infection as discussed above.

Interdependence of KEGG pathways widely exist and most of these are interrelated with each other via shared components, forming a signaling network to allow for pathway crosstalk. To investigate these interactions, we extracted the DE transcripts shared by multiple enriched pathways and constructed a sketch of the hypothetical pathways network that are significantly altered by QPX infection (Fig. 6). In this framework, M. mercenaria response to QPX infection was initiated upon the sensing of danger signals via cell membrane receptors. The signals subsequently transmitted down through the MAPK, Wnt and Notch pathways and triggered the production of a series of host defense factors as the end results. In parallel, activation of pathways regulating actin cytoskeleton and leukocyte transendothelial migration facilitated the recruitment of hemocytes to the infection area to build a barrier of cellular defense against the parasite. Recruited hemocytes then attached to and encapsulated QPX cells as suggested by the modulation of focal adhesion and ECM receptor interaction pathways. These cellular activities were performed under a tight regulation of the apoptosis pathway to determine cell fates, resulting in either the survival or death of M. mercenaria cells.

\section{Distinctive transcriptomic pattern of healthy clams}

A suite of transcripts (407, Fig. 4, Additional file 4 and Table 5) exhibited higher transcription levels in healthy 
Table 4 Selected transcripts with annotated functions (GO terms) related to immune response that were over-expressed during $M$. mercenaria systemic response against QPX. Additional information on these transcripts is given in Additional file 3. "Inf" designates an infinite fold change calculated for focal response as the expression of that transcript in non-nodule tissue was equal to 0

\begin{tabular}{|c|c|c|c|c|}
\hline Transcripts ID & Annotation & Regul-ation & Fold change & Function/GOs \\
\hline \multicolumn{5}{|l|}{ Stress protein } \\
\hline comp39934_c1_seq1 & heat shock $70 \mathrm{kda}$ protein & Up & Inf & P:response to stress \\
\hline comp181704_c3_seq49 & heat shock $70 \mathrm{kda}$ protein $12 \mathrm{~b}$ & Up & 452.9 & - \\
\hline comp68505_c0_seq1 & heat shock protein 70 & Up & 37.8 & P:response to stress \\
\hline comp38810_c0_seq1 & hsp90 family member & Up & 64.7 & P:response to stress \\
\hline comp192296_c2_seq3 & usp-like protein isoform 2 & Up & 46.2 & P:response to stress \\
\hline \multicolumn{5}{|l|}{ Immune effectors } \\
\hline comp192209_c0_seq3 & hemocyte defensin partial & Up & 173.5 & P:defense response \\
\hline comp186386_c5_seq2 & c-type lysozyme 2 & Up & 20.0 & - \\
\hline comp186386_c5_seq1 & c-type lysozyme 2 & Up & $\operatorname{lnf}$ & - \\
\hline comp164821_c0_seq2 & clq domain containing protein & Up & 8.3 & Lectin \\
\hline comp164821_c0_seq3 & clq domain containing protein & Up & 11.9 & Lectin \\
\hline comp190576_c0_seq27 & $\begin{array}{l}\text { low affinity immunoglobulin } \\
\text { epsilon fc receptor }\end{array}$ & Up & 134.1 & F:carbohydrate binding; Lectin \\
\hline comp190576_c0_seq26 & $\begin{array}{l}\text { low affinity immunoglobulin } \\
\text { epsilon fc receptor }\end{array}$ & Up & 201.6 & F:carbohydrate binding; Lectin \\
\hline comp191987_c0_seq10 & macrophage mannose receptor 1-like & Up & 7.8 & F:carbohydrate binding; Lectin \\
\hline comp189507_c1_seq9 & ferric-chelate reductase 1 & Up & 10.7 & Iron transport \\
\hline \multicolumn{5}{|l|}{ Peptidases } \\
\hline comp156268_c1_seq1 & cathepsin k-like & Up & 9.5 & - \\
\hline comp184090_c0_seq6 & calpain 11-like & Up & 10.1 & - \\
\hline comp189096_c0_seq4 & counting factor associated protein d-like & Up & 36.1 & F:cysteine-type peptidase activity \\
\hline comp176418_c0_seq1 & isoaspartyl peptidase & Up & 8.0 & F:hydrolase activity \\
\hline \multicolumn{5}{|l|}{ Anti-apoptotic factors } \\
\hline comp178822_c0_seq1 & interleukin IL17-like & Up & 11.6 & P:inflammatory response; F:cytokine activity \\
\hline comp176786_c2_seq3 & deoxyguanosine mitochondrial & Up & 29.5 & $\begin{array}{l}\text { F:tumor necrosis factor receptor } \\
\text { binding; P:immune response; }\end{array}$ \\
\hline comp192603_c1_seq8 & $\begin{array}{l}\text { baculoviral iap repeat- } \\
\text { containing protein 7-a }\end{array}$ & Up & 10.3 & P:negative regulation of peptidase activity \\
\hline comp192603_c1_seq7 & $\begin{array}{l}\text { baculoviral iap repeat- } \\
\text { containing protein } 7 \text {-a }\end{array}$ & Up & 14.3 & P:negative regulation of peptidase activity \\
\hline comp191786_c0_seq3 & $\begin{array}{l}\text { baculoviral iap repeat- } \\
\text { containing protein partial }\end{array}$ & Up & 8.9 & - \\
\hline
\end{tabular}

clams as compared to diseased clams (considering both nodule and non-nodule tissues). A considerable fraction of these transcripts were related to metabolic processes, nucleic acids binding and transcriptional regulation. The over-expressed immune-related transcripts identified in healthy clams are of particular interest as they may be involved in $M$. mercenaria resistance towards QPX. For example, an antimicrobial protein (aplysianin A [125, 126]) was exclusively identified in healthy clams with almost no detection in diseased clams. In addition, the highest expression levels of a serine protease inhibitor were also observed in healthy clams. A serine protease inhibitor has been linked to oyster (Crassostrea virginica) resistance against the protozoan parasite Perkinsus marinus [103-106, 127]. Therefore, the high expression of the serine protease inhibitor in healthy clams supports its involvement in $M$. mercenaria's resistance against QPX, likely by inhibiting the activity of parasite proteases. Moreover, a pathogen recognition protein (ctype lectin domain family 10 member A-like) was significantly higher in healthy clams as compared to diseased animals, which may also contribute to clam resistance against QPX by promoting microbial recognition and encapsulation [128]. Previous studies demonstrated that clam genetic background affects $M$. mercenaria resistance toward QPX [4, 129, 130], therefore immune- 


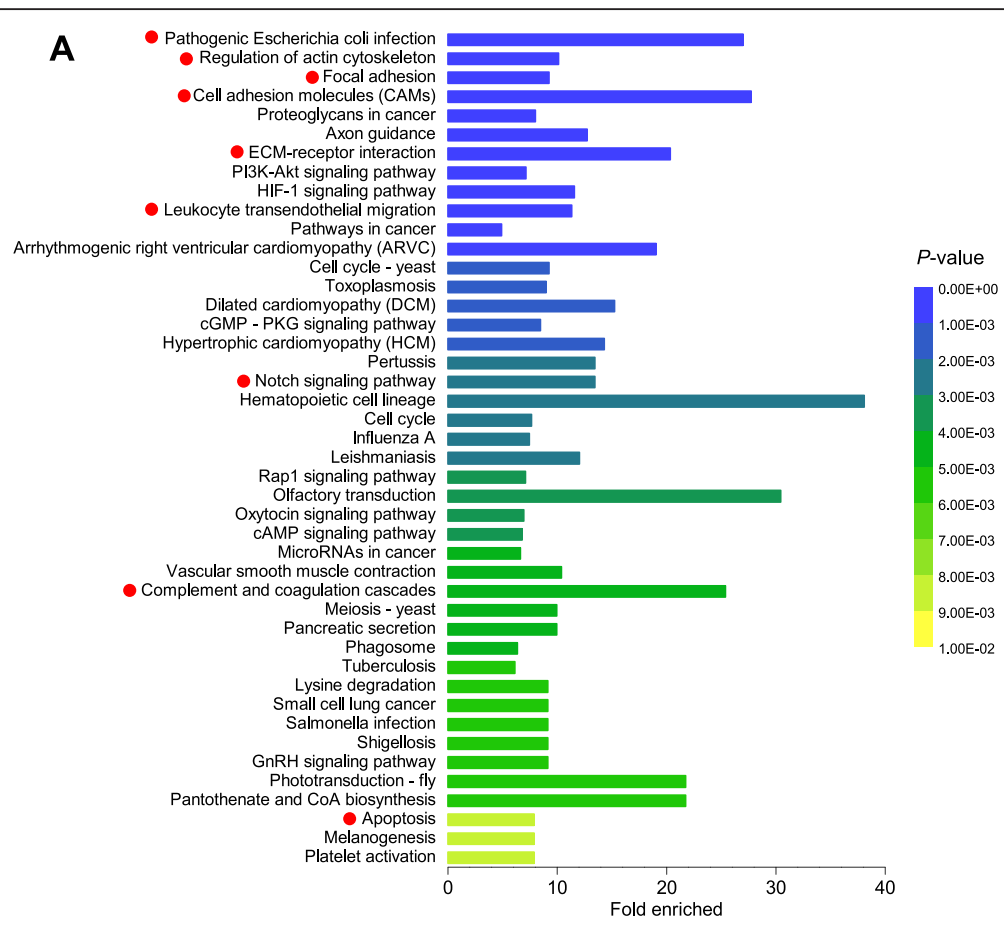

B

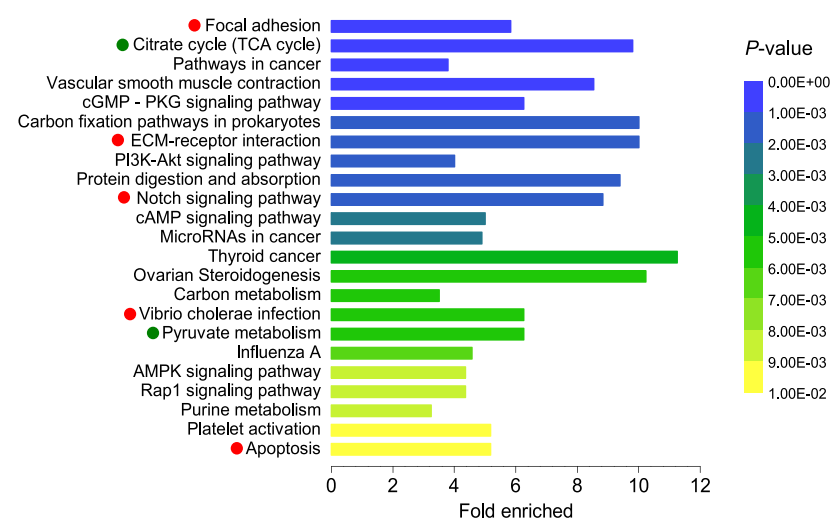

Fig. 5 Significantly enriched KEGG pathways in M. mercenaria derived from the differentially expressed (DE) genes during focal (a) and systemic (b) response against QPX. The KEGG pathways having significant enrichment $(P<0.01)$ are presented, and the bar shows the $x$-fold enrichment of each KEGG pathway. Pathways involved in immune response are marked with red dots while metabolism-related pathways are marked with green dots

related transcripts specifically associated with healthy clams represent excellent candidates as molecular markers for further research on screening and breeding QPX-resistant $M$. mercenaria strains.

On the other hand, a subset of transcripts (126, Fig. 4 and Table 5) exhibited lowest expression levels in healthy clams. These include stress protein HSP 70 and USP, as well as the detoxification molecule cytochrome p450, which together highlight the stress experienced by clams as the result of QPX infection [63, 131-135]. In addition, other immune related transcripts including a protease (isoaspartyl peptidase/L-asparaginase, ASRGL), a protease inhibitor (GTP-binding protein yptV4), a Ctype lectin (MRC1), and molecules involved in tissue regeneration and cell signaling, were also underexpressed in healthy clams as compared to infected individuals, suggesting their role in fighting the infection.

\section{Conclusions}

This is one of the first studies contrasting focal and systemic immune responses to infections in invertebrates using high-throughput sequencing. Resulting transcriptome represents a significant addition to the so far limited public genomic information available for $M$. mercenaria. The transcriptomic profiles of healthy and infected clams reflected complex interactions between the host immune system and the pathogen leading to molecular changes at both the infection foci and the 


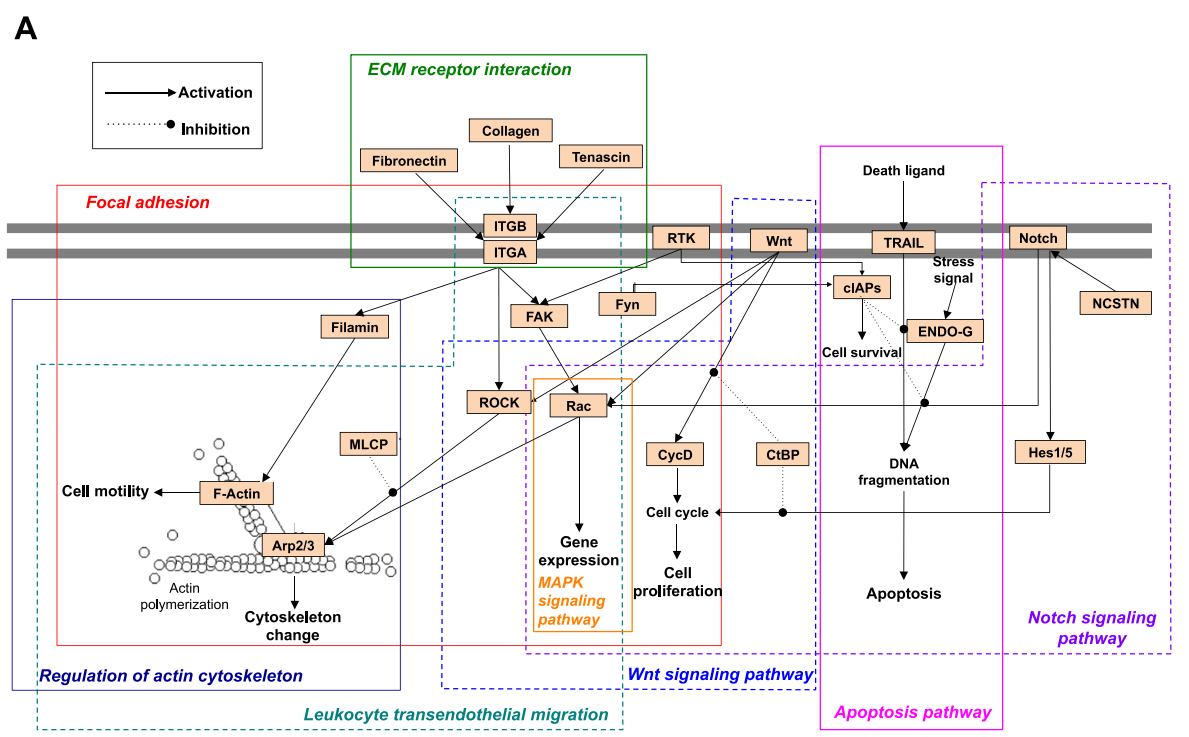

B
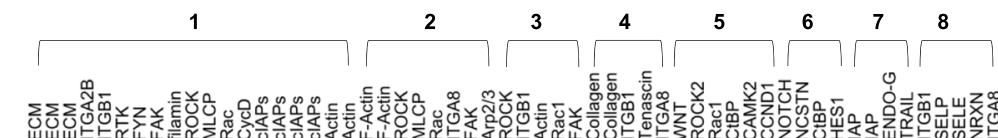

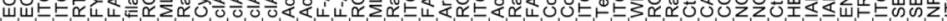

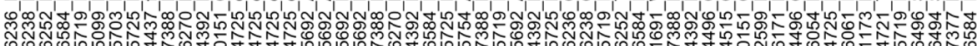

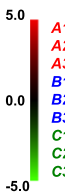

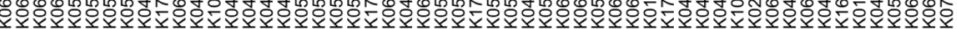

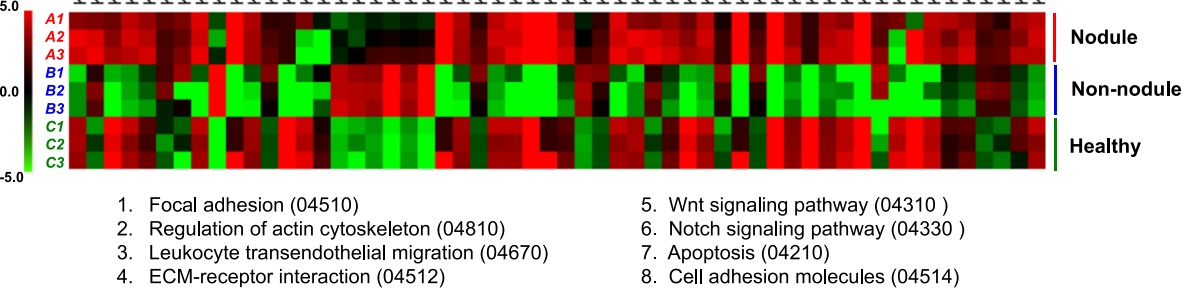

Fig. 6 Overview of immune-related enriched pathways of differentially expressed (DE) transcripts during M. mercenaria response to QPX. a Schematic diagram of enriched pathways and their interactions. Only pathway components encoded by DE transcripts (shown in text boxes) are presented within each enriched KEGG pathway (framed in orthogonal polygons). Arrows display possible interactions (e.g., activation, inhibition) between pathway components. b Overview of DE transcripts expression associated with enriched pathways in nodule, non-nodule and healthy M. mercenaria tissues. The red and green heatmap values indicate $\log _{2}$ fold of relative expression levels for individual transcripts. Arp2/3: actin related protein 2/3 complex; CtBP: C-terminal binding protein; CAMK2: calcium/calmodulin-dependent protein kinase (CaM kinase) II; CycD: cyclin D1 (CCND1); ECM: von Willebrand factor; ENDO-G: endonuclease G; FAK: focal adhesion kinase; FYN: tyrosine-protein kinase; HES1: hairy and enhancer of split 1; IAP: baculoviral IAP repeat-containing protein, CIAPs; ITGA: intergrin alpha; ITGB: intergrin beta; MLCP: serine/threonine-protein phosphatase PP1 catalytic subunit; NRXN: neurexin; NCSTN: nicastrin; Rac: Ras-related C3 botulinum toxin substrate; ROCK: Rho-associated protein kinase; RTK: proto-oncogene tyrosine-protein kinase; SELE: selectin, endothelial cell; SELP: selectin, platelet; TRAIL: tumor necrosis factor ligand superfamily member 10); WNT: wingless-type MMTV integration site family

systemic level. In general, the systemic responses of $M$. mercenaria reflected prevailing transcriptomic suppression accompanied with a contrasting over-expression of stress proteins and soluble antimicrobial effectors; whereas the focal response highlighted cell-cell interactions between hemocytes and the parasite that typically result in local inflammation, extracellular degradation, encapsulation, granuloma formation, and wound repair. What needs to be kept in mind is that the regulation of these genes can be the result of either an effective immune response or a symptom of a future death. In fact, several apoptotic and anti-apoptotic genes were regulated. This highlights a fine adjustment of $M$. mercenaria defense mechanisms to precisely adapt to the infection development (e.g., through the modulation of energy allocation, apoptotic and antiapoptotic processes and mobilization of different signaling pathways). In addition, the identification of immune-related transcripts that were particularly associated with healthy clams offered new perspectives on the molecular features putatively involved in clam resistance against QPX. 
Table 5 Selected transcripts with annotated functions (GO terms) related to immune response that were differentially expressed in naïve M. mercenaria as compared to QPX infected individuals. Additional information on these transcripts is given in Additional file 4. "Inf" designates an infinite fold change calculated for focal response as the expression of that transcript in non-nodule tissue was equal to 0

\begin{tabular}{llll}
\hline Transcripts ID & Annotation & Fold change & Function/GOs \\
\hline Over-expressed & & 84.0 & - \\
comp168250_c0_seq2 & macrophage expressed protein & 18.1 & F:protein binding \\
comp160023_c0_seq1 & serine protease inhibitor 1 & 148.6 & P:response to biotic stimulus \\
comp117137_c0_seq1 & proline-rich transmembrane protein 1 & 121.4 & F:carbohydrate binding \\
comp134883_c0_seq1 & c-type lectin domain family 10 member a-like & Inf & P:defense response; F:oxidoreductase activity \\
comp180146_c3_seq31 & aplysianin a precursor & 20.0 & F:scavenger receptor activity \\
comp179365_c0_seq7 & insulin-related peptide receptor & 16.2 & P:apoptotic process; \\
comp187368_c2_seq9 & apoptosis 1 inhibitor & 391.0 & P:negative regulation of apoptotic process; \\
comp193015_c0_seq1 & baculoviral iap repeat-containing protein 4 & & F:ubiquitin-protein ligase activity \\
Under-expressed & & Inf & P:response to stress \\
comp39934_c1_seq1 & heat shock 70 kda protein & -46.2 & P:response to stress \\
comp192296_c2_seq3 & usp-like protein isoform 2 & -75.1 & - \\
comp169961_c0_seq2 & cytochrome p450 1a1 & -8.0 & -7.8 \\
comp176418_c0_seq1 & isoaspartyl peptidase l-asparaginase-like & -9.8 & F:hydrolase activity \\
comp191987_c0_seq10 & macrophage mannose receptor 1-like & F:carbohydrate binding \\
comp186563_c0_seq7 & bile salt-activated lipase-like & F:hydrolase activity
\end{tabular}

\section{Methods}

\section{Clam tissue and RNA samples preparation}

Adult hard clams $(54 \pm 5 \mathrm{~mm}$ in length, mean \pm standard deviation) were collected from a QPX enzootic area in Massachusetts. Clams were shucked and grossly examined for the presence of nodules along the mantle rim. Nodule tissues were dissected and a small piece of each nodule was microscopically examined (fresh mount) for the identification of QPX cells. Aliquot tissues from positive nodules were submitted to RNA extraction. Meanwhile, a piece of seemingly healthy tissue that is anatomically symmetrical to the nodule was collected from the same clam (e.g., "healthy" tissue from a diseased clam) and divided into 2 aliquots, with the first aliquot used for QPX detection by qPCR [10] and the second used for RNA extraction. Mantle tissues were also collected from "seemingly" healthy clams (no visible nodules) and used for QPX detection and RNA extraction. Following confirmation of disease status by qPCR, the samples were divided into the following 3 categories: (1) infected tissue from a diseased clam, (2) "healthy"(non-nodule) tissue from a diseased clam, and (3) "healthy" tissue from a healthy clam. Total RNA was individually extracted using TRIzol ${ }^{\circ}$ Reagent (Invitrogen, Carlsbad, CA, USA). Further RNA clean-up and oncolumn DNase digestion were performed with RNeasy Mini Kit (Qiagen Ltd., Crawley, UK) according to the manufacturer's guideline. RNA quantity and quality were analyzed on Nanodrop ND-1000 (Thermo Scientific, Wilmington, USA). Only RNA samples with absorption ratios of A260/A280 close to 2.0 were used for RNA-seq analysis. A total amount of $3 \mu \mathrm{g}$ RNAs per sample was used and pooled into a total of 3 pools ( 3 clams per pool) representing each tissue category (9 pools in all). The pooling strategy is shown in Table 1.

\section{RNA sequencing, de novo assembly and annotation}

The sequencing of each pooled RNA sample as a paired end (PE) reads library (100 pb) was performed on Illumina HiSeq 2000 platform at the McGill University and Genome Quebec Innovation Center (Montreal, Canada), producing from 27.1 up to 46.8 millions of PE reads per sample (Table 1). Raw reads were filtered and trimmed according to length and quality score (min length $60 \mathrm{nt}$, end trimming quality 25 , min quality filtering: 20 on $75 \%$ of the read length) using the FASTX-Toolkit software v 0.0.13 (http://hannonlab.cshl.edu/fastx_toolkit/). rRNA cleaning was performed using the riboPicker software v 4.0.3 (http://ribopicker.sourceforge.net) [136] against SILVA database v111. And finally pair retrieval was performed using a homemade python script accessible through GitHub (https://github.com/ppericard/bioinfotoolkit). High quality filtered sequence reads from all libraries were combined and subsequently used for de novo assembly. Assembly was based on the de Bruijn graph assembler Trinity (http://trinityrnaseq.sourceforge.net/) [137] 
using the default parameters. Assembly quality was controlled by re-mapping raw reads back to the transcripts using bowtie 2 and RSEM within Trinity package scripts. The assembled transcripts with sequence length longer than 200 bp, re-mapping FPKM (fragments per kilobase of transcript per million mapped reads) greater than 1 and isoform discovery level greater than $1 \%$ were then considered for the following annotation and transcript abundance quantitation. Annotation of this de novo assembled transcriptome was performed using Blast2GO (http:// www.blast2go.com) with a semi-automated functional annotation based on sequence homology search. Putative gene identities was obtained by Blastx search against $\mathrm{Na}$ tional Center for Biotechnology Information (NCBI) nonredundant sequences (nr) database with the E-value threshold setting at $1 \mathrm{E}-03$. Putative gene functions were predicted by sequence similarity search against Gene Ontology (GO, http://www.geneontology.org/) database and assigning GO annotation terms to each mapped transcript. Protein domain search and enzyme annotation were also performed using InterPro scan and the Kyoto Encyclopedia of Genes and Genomes (KEGG). KEGG Orthology (KO) terms and KEGG pathways were also assigned to the assembled sequences using the online KEGG Automatic Annotation Server (KAAS, http://www.genome.jp/kegg/kaas/) using bidirectional best-hit methods [138]. This server provides KO annotation and pathway mapping.

\section{Differential gene expression analysis}

The consensus transcriptome generated in the previous steps was used as a reference for transcript abundance analysis. Reads from each single library were separately mapped to this reference transcriptome using the "RNASeq by Expectation-Maximization (RSEM)" method that is bundled within the Trinity package. The expression level of each transcript was determined as the total mapped reads count. The differences in gene expression between clam tissue samples (nodule, non-nodule and healthy tissues) were estimated using the DESeq Bioconductor package (https://github.com/Bioconductor-mirror/SESeq/tree/release-3.2) [139] in R statistical software (R Development Core Team, 2010; http://www.R-project.org). The threshold for defining significant differentially expressed (DE) transcripts between two different conditions (3 replications in each condition) was set as adjusted $p$-value smaller than 0.001 and absolute $\log _{2}$ (fold change) values greater than 2. Expression patterns of DE transcripts were also analyzed by a K-means clustering method using Euclidean distance based on expression levels over all input samples. For further analysis, only those DE transcripts with annotation were considered as candidates of interest and were subsequently divided into over- and under-expressed groups.

\section{Availability of supporting data}

The data sets supporting the results of this study are available at the NCBI short Read Archive database under the SRA accession number SRP068241.

\section{Additional files}

\begin{abstract}
Additional file 1: Sequence summary of M. mercenaria de novo assembled transcriptome. This is a Microsoft Excel worksheet that contains descriptions and annotation information of individual transcript sequences. (XLSX $6600 \mathrm{~kb}$ )

Additional file 2: Differentially expressed (DE) sequences associated with $M$. mercenaria focal response against QPX. This file is a Microsoft Excel worksheet that contains separate lists of over- and under-expressed transcripts with their expression levels and fold change values given. (XLSX $143 \mathrm{~kb}$ )

Additional file 3: Differentially expressed (DE) sequences associated with $M$. mercenaria systemic response against QPX. This file is a Microsoft Excel worksheet that contains separate lists of over- and under-expressed transcripts with their expression levels and fold change values given. (XLSX $327 \mathrm{~kb}$ )

Additional file 4: Transcripts that were differentially expressed in naïve M. mercenaria as compared to QPX infected individuals. This file is a Microsoft Excel worksheet that contains separate lists of over- and underexpressed transcripts with their expression levels and fold change values given. (XLSX $128 \mathrm{~kb}$ )
\end{abstract}

\section{Competing interests}

The authors declare that they have no competing interests.

\section{Authors' contributions}

BA and EPE designed the study, coordinated experiments and supervised data analysis and manuscript drafting. KW carried out the experiments, analyzed the data and drafted the manuscript. EC participated in data analysis and helped in drafting the methodology section of the manuscript. CDC helped with data analysis. All authors read and approved the final manuscript.

\section{Acknowledgments}

This research was supported by projects R/XG-19 and R/FBM-34 (to BA and EPE), funded under award NA07OAR4170010 from the National Sea Grant College Program of NOAA to the Research Foundation of State University of New York on behalf of New York Sea Grant. The study was also partially supported by project IOS 1050596 (to BA and EPE) supported by the National Science Foundation. The statements, findings, conclusions, views and recommendations are those of the authors and do not necessarily reflect the views of any of those organizations.

\section{Author details}

${ }^{1}$ School of Marine and Atmospheric Sciences, Stony Brook University, Stony Brook, NY 11794-5000, USA. ${ }^{2}$ Analyses and Bioinformatics for Marine Science, Station Biologique de Roscoff, 29688 Roscoff Cedex, France.

Received: 6 October 2015 Accepted: 17 February 2016

Published online: 27 February 2016

\section{References}

1. Whyte SK, Cawthorn RJ, McGladdery SE. QPX (Quahaug Parasite X), a pathogen of northern quahaug Mercenaria mercenaria from the gulf of St-Lawrence, Canada. Dis Aquat Organ. 1994;19(2):129-36.

2. Smolowitz R, Leavitt $D$, Perkins F. Observations of a protistan disease similar to QPX in Mercenaria mercenaria (hard clams) from the coast of Massachusetts. J Invertebr Pathol. 1998;71(1):9-25.

3. Anderson RS, Kraus BS, McGladdery SE, Reece KS, Stokes NA. A thraustochytrid protist isolated from Mercenaria mercenaria: molecular characterization and host defense responses. Fish Shellfish Immun. 2003;15(3):183-94. 
4. Ford SE, Kraeuter JN, Barber RD, Mathis G. Aquaculture-associated factors in QPX disease of hard clams: density and seed source. Aquaculture. 2002; 208(1-2):23-38.

5. Ragone Calvo LM, Walker JG, Burreson EM. Prevalence and distribution of QPX, Quahog Parasite Unknown, in hard clams Mercenaria mercenaria in Virginia, USA. Dis Aquat Organ. 1998;33(3):209-19.

6. MacCallum GS, McGladdery SE. Quahog Parasite Unknown (QPX) in the northern quahog Mercenaria mercenaria (Linnaeus, 1758) and M. mercenaria var. notata from Atlantic Canada, survey results from three maritime provinces. J Shellfish Res. 2000;19(1):43-50.

7. Dove ADM, Bowser PR, Cerrato RM. Histological Analysis of an Outbreak of QPX Disease in Wild Hard Clams Mercenaria mercenaria in New York. J Aquat Anim Health. 2004;16(4):246-50.

8. Lyons MM, Ward JE, Smolowitz R, Uhlinger KR, Gast RJ. Lethal marine snow: Pathogen of bivalve mollusc concealed in marine aggregates. Limnol Oceanogr. 2005;50(6):1983-8.

9. Gast RJ, Moran DM, Audemard C, Lyons MM, DeFavari J, Reece KS, et al. Environmental distribution and persistence of Quahog Parasite Unknown (QPX). Dis Aquat Organ. 2008;81(3):219-29.

10. Liu QQ, Allam B, Collier JL. Quantitative Real-Time PCR Assay for QPX (Thraustochytriidae), a Parasite of the Hard Clam (Mercenaria mercenaria). Appl Environ Microb. 2009;75(14):4913-8.

11. Perrigault M, Dahl SF, Espinosa EP, Gambino L, Allam B. Effects of temperature on hard clam (Mercenaria mercenaria) immunity and QPX (Quahog Parasite Unknown) disease development: II. Defense parameters. J Invertebr Pathol. 2011;106(2):322-32

12. Bayne CJ. Phagocytosis and non-self recognition in invertebrates. Bioscience. 1990;40(10):723-31.

13. Canesi L, Gallo G, Gavioli M, Pruzzo C. Bacteria-hemocyte interactions and phagocytosis in marine bivalves. Microsc Res Tech. 2002;57(6):469-76.

14. Perrigault M, Dahl SF, Espinosa EP, Allam B. Effects of salinity on hard clam (Mercenaria mercenaria) defense parameters and QPX disease dynamics. J Invertebr Pathol. 2012;110(1):73-82.

15. Perrigault $M$, Tanguy A, Allam B. Identification and expression of differentially expressed genes in the hard clam, Mercenaria mercenaria, in response to quahog parasite unknown (QPX). Bmc Genomics. 2009;10:377.

16. Perrigault $M$, Allam B. Differential immune response in the hard clam (Mercenaria mercenaria) against bacteria and the protistan pathogen QPX (quahog parasite unknown). Fish Shellfish Immun. 2012;32(6):1124-34.

17. Leite RB, Milan M, Coppe A, Bortoluzzi S, dos Anjos A, Reinhardt R, et al. mRNA-Seq and microarray development for the Grooved carpet shell clam, Ruditapes decussatus: a functional approach to unravel host -parasite interaction. Bmc Genomics. 2013;14(1):741.

18. Zhao X, Yu H, Kong L, Li Q. Transcriptomic Responses to Salinity Stress in the Pacific Oyster Crassostrea gigas. PLoS ONE. 2012;7(9):e46244.

19. Teaniniuraitemoana V, Huvet A, Levy P, Klopp C, Lhuillier E, Gaertner-Mazouni $\mathrm{N}$, et al. Gonad transcriptome analysis of pearl oyster Pinctada margaritifera: identification of potential sex differentiation and sex determining genes. Bmc Genomics. 2014;15(1):491

20. Adema CM, Hanington PC, Lun C-M, Rosenberg GH, Aragon AD, Stout BA, et al. Differential transcriptomic responses of Biomphalaria glabrata (Gastropoda, Mollusca) to bacteria and metazoan parasites, Schistosoma mansoni and Echinostoma paraensei (Digenea, Platyhelminthes). Mol Immunol. 2010;47(4):849-60.

21. Wang JP, Lindsay BG, Leebens-Mack J, Cui L, Wall K, Miller WC, et al. EST clustering error evaluation and correction. Bioinformatics. 2004:20(17):2973-84

22. Mittapalli O, Bai X, Mamidala P, Rajarapu SP, Bonello P, Herms DA. Tissue-Specific Transcriptomics of the Exotic Invasive Insect Pest Emerald Ash Borer (Agrilus planipennis). PLOS ONE. 2010;5(10):e13708.

23. Pelechano $V$, Wei W, Steinmetz LM. Extensive transcriptional heterogeneity revealed by isoform profiling. Nature. 2013:497(7447):127-31.

24. Luo W, Friedman MS, Shedden K, Hankenson KD, Woolf PJ. GAGE: generally applicable gene set enrichment for pathway analysis. BMC Bioinformatics. 2009;10(1):161

25. Humphries JE, Yoshino TP. Cellular receptors and signal transduction in molluscan hemocytes: connections with the innate immune system of vertebrates. Integr Comp Biol. 2003;43(2):305-12.

26. Soudant P, E Chu FL, Volety A. Host-parasite interactions: Marine bivalve molluscs and protozoan parasites, Perkinsus species. J Invertebr Pathol. 2013;114(2):196-216
27. Canesi L, Betti M, Ciacci C, Lorusso LC, Pruzzo C, Gallo G. Cell signalling in the immune response of mussel hemocytes. Invertebrate Survival Journal: ISJ. 2006;3:40-9.

28. Hatanaka R, Sekine Y, Hayakawa T, Takeda K, Ichijo H. Signaling pathways in invertebrate immune and stress response. Invertebrate Survival Journal: ISJ. 2009:6:32-43.

29. Jeannin $P$, Jaillon S, Delneste Y. Pattern recognition receptors in the immune response against dying cells. Curr Opin Immunol. 2008;20(5):530-7.

30. Wang L, Wang L, Zhang D, Li F, Wang M, Huang M, et al. A novel C-type lectin from crab Eriocheir sinensis functions as pattern recognition receptor enhancing cellular encapsulation. Fish Shellfish Immun. 2013;34(3):832-42.

31. Vazquez-Mendoza A, Carrero JC, Rodriguez-Sosa M. Parasitic infections: a role for C-type lectins receptors. Biomed Res Int. 2013;2013:456352.

32. Yang J, Qiu L, Wei X, Wang L, Wang L, Zhou Z, et al. An ancient C-type lectin in Chlamys farreri (CfLec-2) that mediate pathogen recognition and cellular adhesion. Dev Comp Immunol. 2010;34(12):1274-82.

33. Wang $L$, Wang $L$, Yang J, Zhang $H$, Huang $M$, Kong $P$, et al. A multi-CRD Ctype lectin with broad recognition spectrum and cellular adhesion from Argopecten irradians. Dev Comp Immunol. 2012;36(3):591-601.

34. Ao J, Ling E, Yu XQ. Drosophila C-type lectins enhance cellular encapsulation. Mol Immunol. 2007:44(10):2541-8.

35. Lasky LA. Selectin-Carbohydrate Interactions and the Initiation of the Inflammatory Response. Annu Rev Biochem. 1995;64(1):113-40.

36. Gazi $U$, Martinez-Pomares $L$. Influence of the mannose receptor in host immune responses. Immunobiology. 2009;214(7):554-61.

37. Moreira R, Milan M, Balseiro P, Romero A, Babbucci M, Figueras A, et al. Gene expression profile analysis of Manila clam (Ruditapes philippinarum) hemocytes after a Vibrio alginolyticus challenge using an immune-enriched oligo-microarray. Bmc Genomics. 2014;15(1):267.

38. Gordon S. Pattern recognition receptors: doubling up for the innate immune response. Cell. 2002;111(7):927-30.

39. Liu L, Yang J, Qiu L, Wang L, Zhang $H$, Wang $M$, et al. A novel scavenger receptor-cysteine-rich (SRCR) domain containing scavenger receptor identified from mollusk mediated PAMP recognition and binding. Dev Comp Immunol. 2011;35(2):227-39.

40. Mukhopadhyay S, Gordon S. The role of scavenger receptors in pathogen recognition and innate immunity. Immunobiology. 2004;209(1-2):39-49.

41. Blumbach B, Pancer Z, Diehl-Seifert B, Steffen R, Munkner J, Muller I, et al. The putative sponge aggregation receptor. Isolation and characterization of a molecule composed of scavenger receptor cysteine-rich domains and short consensus repeats. J Cell Sci. 1998;111(Pt 17):2635-44.

42. van der Laan LJ, Dopp EA, Haworth R, Pikkarainen T, Kangas M, Elomaa O, et al. Regulation and functional involvement of macrophage scavenger receptor MARCO in clearance of bacteria in vivo. J Immunol. 1999;162(2):939-47.

43. Bowdish DM, Gordon S. Conserved domains of the class A scavenger receptors: evolution and function. Immunol Rev. 2009;227(1):19-31.

44. Rast JP, Smith LC, Loza-Coll M, Hibino T, Litman GW. Genomic insights into the immune system of the sea urchin. Science. 2006;314(5801):952-6.

45. Zhang Y, He X, Yu F, Xiang Z, Li J, Thorpe KL, et al. Characteristic and functional analysis of toll-like receptors (TLRs) in the lophotrocozoan, Crassostrea gigas, reveals ancient origin of TLR-mediated innate immunity. PLoS One. 2013;8(10):e76464.

46. Qiu L, Song L, Xu W, Ni D, Yu Y. Molecular cloning and expression of a Toll receptor gene homologue from Zhikong Scallop, Chlamys farreri. Fish Shellfish Immunol. 2007;22(5):451-66.

47. Moreira R, Balseiro P, Planas JV, Fuste B, Beltran S, Novoa B, et al. Transcriptomics of in vitro immune-stimulated hemocytes from the Manila clam Ruditapes philippinarum using high-throughput sequencing. PLoS One. 2012;7(4):e35009.

48. Mateo DR, Greenwood SJ, Araya MT, Berthe FC, Johnson GR, Siah A. Differential gene expression of gamma-actin, Toll-like receptor 2 (TLR-2) and interleukin-1 receptor-associated kinase 4 (IRAK-4) in Mya arenaria haemocytes induced by in vivo infections with two Vibrio splendidus strains. Dev Comp Immunol. 2010;34(7):710-4

49. Akhouayri I, Turc C, Royet J, Charroux B. Toll-8/Tollo Negatively Regulates Antimicrobial Response in the Drosophila Respiratory Epithelium. Plos Pathog. 2011;7(10):e1002319.

50. Williams GT, Williams WJ. Granulomatous inflammation-a review. J Clin Pathol. 1983;36(7):723-33.

51. de Brito T, Franco MF. Granulomatous inflammation. Revista do Instituto de Medicina Tropical de Sao Paulo. 1994;36(2):185-92.

52. Sun $L$, Ye RD. Role of $G$ protein-coupled receptors in inflammation. Acta pharmacologica Sinica. 2012;33(3):342-50. 
53. Cho H, Kehrl JH. Regulation of immune function by $\mathrm{G}$ protein-coupled receptors, trimeric G proteins, and RGS proteins. Prog Mol Biol Transl Sci. 2009;86:249-98.

54. Zhang $Y$, Wang $H$. Integrin signalling and function in immune cells. Immunology. 2012;135(4):268-75.

55. Stavitsky AB. Regulation of granulomatous inflammation in experimental models of schistosomiasis. Infect Immun. 2004;72(1):1-12.

56. Ha EM, Oh CT, Bae YS, Lee WJ. A direct role for dual oxidase in Drosophila gut immunity. Science. 2005;310(5749):847-50

57. Bae YS, Choi MK, Lee WJ. Dual oxidase in mucosal immunity and hostmicrobe homeostasis. Trends Immunol. 2010;31(7):278-87.

58. Inada M, Kihara K, Kono T, Sudhakaran R, Mekata T, Sakai M, et al. Deciphering of the Dual oxidase (Nox family) gene from kuruma shrimp, Marsupenaeus japonicus: Full-length cDNA cloning and characterization. Fish Shellfish Immun. 2013;34(2):471-85.

59. Libro S, Kaluziak ST, Vollmer SV. RNA-seq Profiles of Immune Related Genes in the Staghorn Coral Acropora cervicornis Infected with White Band Disease. PLOS ONE. 2013;8(11):e81821.

60. Bignon M, Pichol-Thievend C, Hardouin J, Malbouyres M, Brechot N, Nasciutti L, et al. Lysyl oxidase-like protein-2 regulates sprouting angiogenesis and type IV collagen assembly in the endothelial basement membrane. Blood. 2011; 118(14):3979-89.

61. Chang NS, Hsu LJ, Lin YS, Lai FJ, Sheu HM. WW domain-containing oxidoreductase: a candidate tumor suppressor. Trends Mol Med. 2007; 13(1):12-22.

62. Yang Z, Zhao T, Liu Y. Upregulation of tumor suppressor WWOX promotes immune response in glioma. Cell Immunol. 2013;285(1-2):1-5.

63. Behrendt L, Jonsson ME, Goldstone JV, Stegeman JJ. Induction of cytochrome P450 1 genes and stress response genes in developing zebrafish exposed to ultraviolet radiation. Aquat Toxicol. 2010;98(1):74-82.

64. Pan L, Liu N, Xu C, Miao J. Identification of a novel P450 gene belonging to the CYP4 family in the clam Ruditapes philippinarum, and analysis of basaland benzo(a)pyrene-induced mRNA expression levels in selected tissues. Environ Toxicol Pharmacol. 2011;32(3):390-8.

65. Lotze MT, Zeh HJ, Rubartelli A, Sparvero LJ, Amoscato AA, Washburn NR, et al. The grateful dead: damage-associated molecular pattern molecules and reduction/oxidation regulate immunity. Immunol Rev. 2007:220:60-81.

66. Mone $\mathrm{Y}$, Monnin D, Kremer N. The oxidative environment: a mediator of interspecies communication that drives symbiosis evolution. Proc Biol Sci/ The Royal Society. 2014;281(1785):20133112.

67. Terahara K, Takahashi KG. Mechanisms and immunological roles of apoptosis in molluscs. Curr Pharm Des. 2008;14(2):131-7.

68. Sokolova IM. Apoptosis in molluscan immune defense. Invertebrate Survival Journal: ISJ. 2009;6:49-58

69. Gentle IE, Moelter I, Lechler N, Bambach S, Vucikuja S, Hacker G, et al. Inhibitors of apoptosis proteins (IAPs) are required for effective T-cell expansion/survival during antiviral immunity in mice. Blood. 2014;123(5):659-68.

70. Plows LD, Cook RT, Davies AJ, Walker AJ. Phagocytosis by Lymnaea stagnalis haemocytes: A potential role for phosphatidylinositol 3-kinase but not protein kinase A. J Invertebr Pathol. 2006;91(1):74-7.

71. Yoshino TP, Laursen JR. Production of Schistosoma mansoni daughter sporocysts from mother sporocysts maintained in synxenic culture with Biomphalaria glabrata embryonic (Bge) cells. J Parasitol. 1995;81(5):714-22.

72. Newton K, Dixit VM. Signaling in innate immunity and inflammation. Cold Spring Harb Perspect Biol. 2012;4(3). doi: 10.1101/cshperspect.a006049.

73. Toubiana M, Rosani U, Giambelluca S, Cammarata M, Gerdol M, Pallavicini A, et al. Toll signal transduction pathway in bivalves: complete cds of intermediate elements and related gene transcription levels in hemocytes of immune stimulated Mytilus galloprovincialis. Dev Comp Immunol. 2014;45(2):300-12

74. Philipp EER, Kraemer L, Melzner F, Poustka AJ, Thieme S, Findeisen U, et al. Massively Parallel RNA Sequencing Identifies a Complex Immune Gene Repertoire in the lophotrochozoan Mytilus edulis. PLoS ONE. 2012;7(3):e33091.

75. Theodosiou A, Ashworth A. MAP kinase phosphatases. Genome Biol. 2002; 3(7):REVIEWS3009.

76. Grabarek Z. Insights into modulation of calcium signaling by magnesium in calmodulin, troponin $\mathrm{C}$ and related EF-hand proteins. Biochim Biophys Acta (BBA) - Molecular Cell Research. 2011;1813(5):913-21.

77. Liu Y, Schiff M, Serino G, Deng XW, Dinesh-Kumar SP. Role of SCF ubiquitinligase and the COP9 signalosome in the $\mathrm{N}$ gene-mediated resistance response to Tobacco mosaic virus. The Plant cell. 2002;14(7):1483-96.
78. Bech-Otschir D, Seeger M, Dubiel W. The COP9 signalosome: at the interface between signal transduction and ubiquitin-dependent proteolysis. J Cell Sci. 2002;115(Pt 3):467-73.

79. Nezames CD, Deng XW. The COP9 signalosome: its regulation of cullinbased E3 ubiquitin ligases and role in photomorphogenesis. Plant Physiol. 2012;160(1):38-46.

80. Tosello-Trampont AC, Nakada-Tsukui K, Ravichandran KS. Engulfment of apoptotic cells is negatively regulated by Rho-mediated signaling. J Biol Chem. 2003;278(50):49911-9.

81. Krijnen PA, Sipkens JA, Molling JW, Rauwerda JA, Stehouwer CD, Muller A, et al. Inhibition of Rho-ROCK signaling induces apoptotic and nonapoptotic PS exposure in cardiomyocytes via inhibition of flippase. J Mol Cell Cardiol. 2010:49(5):781-90.

82. He X, Zhang Y, Yu Z. An Mpeg (macrophage expressed gene) from the Pacific oyster Crassostrea gigas: molecular characterization and gene expression. Fish Shellfish Immunol. 2011;30(3):870-6.

83. D'Angelo ME, Dunstone MA, Whisstock JC, Trapani JA, Bird PI. Perforin evolved from a gene duplication of MPEG1, followed by a complex pattern of gene gain and loss within Euteleostomi. Bmc Evol Biol. 2012;12(1):59.

84. Staal FJ, Luis TC, Tiemessen MM. WNT signalling in the immune system: WNT is spreading its wings. Nat Rev Immunol. 2008;8(8):581-93.

85. Holstein TW. The evolution of the Wnt pathway. Cold Spring Harb Perspect Biol. 2012;4(7):a007922

86. Zhang Z, Deb A, Zhang Z, Pachori A, He W, Guo J, et al. Secreted frizzled related protein 2 protects cells from apoptosis by blocking the effect of canonical Wnt3a. J Mol Cell Cardiol. 2009;46(3):370-7.

87. Fathke C, Wilson L, Shah K, Kim B, Hocking A, Moon R, et al. Wht signaling induces epithelial differentiation during cutaneous wound healing. Bmc Cell Biol. 2006;7(1):4.

88. Yuan JS, Kousis PC, Suliman S, Visan I, Guidos CJ. Functions of notch signaling in the immune system: consensus and controversies. Annu Rev Immunol. 2010;28:343-65.

89. Rangel JR, Chung Y, Rosenbach M, Ziober AF, Zhang PJ, Schaffer A. Expression of Notch signaling components in cutaneous foreign body and sarcoidal granulomas and fusing macrophages. Am J Dermatopathol. 2014;36(5):409-13.

90. Palaga T, Buranaruk C, Rengpipat S, Fauq AH, Golde TE, Kaufmann SH, et al. Notch signaling is activated by TLR stimulation and regulates macrophage functions. Eur J Immunol. 2008;38(1):174-83.

91. Zhang Q, Wang C, Liu Z, Liu X, Han C, Cao X, et al. Notch signal suppresses Toll-like receptor-triggered inflammatory responses in macrophages by inhibiting extracellular signal-regulated kinase 1/2-mediated nuclear factor kappaB activation. J Biol Chem. 2012;287(9):6208-17.

92. Manoury B, Roghanian A, Sallenave JM. Serine and cysteine proteases and their inhibitors as antimicrobial agents and immune modulators. In: Vergnolle N, Chignard M, editors. Proteases and their receptors in inflammation. Progress in Inflammation Research. Basel: Springer; 2011. p. 27-50.

93. Shpacovitch $V$, Feld M, Hollenberg MD, Luger TA, Steinhoff M. Role of protease-activated receptors in inflammatory responses, innate and adaptive immunity. J Leukoc Biol. 2008;83(6):1309-22.

94. Meyer-Hoffert U, Wiedow O. Neutrophil serine proteases: mediators of innate immune responses. Curr Opin Hematol. 2011;18(1):19-24.

95. Khokha R, Murthy A, Weiss A. Metalloproteinases and their natural inhibitors in inflammation and immunity. Nat Rev Immunol. 2013;13(9):649-65.

96. Murthy A, Shao YW, Narala SR, Molyneux SD, Zuniga-Pflucker JC, Khokha R. Notch activation by the metalloproteinase ADAM17 regulates myeloproliferation and atopic barrier immunity by suppressing epithelial cytokine synthesis. Immunity. 2012;36(1):105-19.

97. Sottrup-Jensen L. Alpha-macroglobulins: structure, shape, and mechanism of proteinase complex formation. J Biol Chem. 1989;264(20):11539-42.

98. Borth W. Alpha 2-macroglobulin, a multifunctional binding protein with targeting characteristics. Faseb J. 1992;6(15):3345-53.

99. Armstrong PB. Proteases and protease inhibitors: a balance of activities in host-pathogen interaction. Immunobiology. 2006;211(4):263-81.

100. Allam B, Pales Espinosa E, Tanguy A, Jeffroy F, Le Bris C, Paillard C. Transcriptional changes in Manila clam (Ruditapes philippinarum) in response to Brown Ring Disease. Fish Shellfish Immunol. 2014;41(1):2-11.

101. Anderson RS, Kraus BS, McGladdery S, Smolowitz R. QPX, a pathogen of quahogs (hard clams), employs mucoid secretions to resist host antimicrobial agents. J Shellfish Res. 2003;22(1):205-8.

102. Rubin E, Tanguy A, Perrigault M, Pales Espinosa E, Allam B. Characterization of the transcriptome and temperature-induced differential gene expression in QPX, the thraustochytrid parasite of hard clams. Bmc Genomics. 2014;15(1):245. 
103. He Y, Yu H, Bao Z, Zhang Q, Guo X. Mutation in promoter region of a serine protease inhibitor confers Perkinsus marinus resistance in the eastern oyster (Crassostrea virginica). Fish Shellfish Immunol. 2012;33(2):411-7.

104. La Peyre JF, Xue QG, Itoh N, Li Y, Cooper RK. Serine protease inhibitor cvSI-1 potential role in the eastern oyster host defense against the protozoan parasite Perkinsus marinus. Dev Comp Immunol. 2010;34(1):84-92.

105. Yu H, He Y, Wang X, Zhang Q, Bao Z, Guo X. Polymorphism in a serine protease inhibitor gene and its association with disease resistance in the eastern oyster (Crassostrea virginica Gmelin). Fish Shellfish Immunol. 2011;30(3):757-62.

106. Xue QG, Waldrop GL, Schey KL, Itoh N, Ogawa M, Cooper RK, et al. A novel slow-tight binding serine protease inhibitor from eastern oyster (Crassostrea virginica) plasma inhibits perkinsin, the major extracellular protease of the oyster protozoan parasite Perkinsus marinus. Comp Biochem Physiol B Biochem Mol Biol. 2006;145(1):16-26.

107. Moné Y, Gourbal B, Duval D, Du Pasquier L, Kieffer-Jaquinod S, Mitta G. A large repertoire of parasite epitopes matched by a large repertoire of host immune receptors in an invertebrate host/parasite model. PLoS Negl Trop Dis. 2010;4(9):e813.

108. Ong ST, Shan Ho JZ, Ho B, Ding JL. Iron-withholding strategy in innate immunity. Immunobiology. 2006;211(4):295-314.

109. Stafford SL, Bokil NJ, Achard ME, Kapetanovic R, Schembri MA, McEwan AG, Sweet MJ. Metal ions in macrophage antimicrobial pathways: emerging roles for zinc and copper. Biosci Rep. 2013;33(4). doi: 10.1042/BSR20130014.

110. Sun $Y$, Zhang $Y$, Fu X, Zhang R, Zou J, Wang S, et al. Identification of two secreted ferritin subunits involved in immune defense of Yesso scallop Patinopecten yessoensis. Fish Shellfish Immunol. 2014;37(1):53-9.

111. Torreilles J, Guerin MC, Roch P. Reactive oxygen species and defense mechanisms in marine bivalves. Cr Acad Sci lii-Vie. 1996;319(3):209-18.

112. Sun Y, Li F, Sun Z, Zhang X, Li S, Zhang C, et al. Transcriptome Analysis of the Initial Stage of Acute WSSV Infection Caused by Temperature Change. PLoS One. 2014;9(3):e90732.

113. Pauletto M, Milan M, Moreira R, Novoa B, Figueras A, Babbucci M, et al. Deep transcriptome sequencing of Pecten maximus hemocytes: a genomic resource for bivalve immunology. Fish Shellfish Immunol. 2014;37(1):154-65.

114. Moya A, Huisman L, Ball EE, Hayward DC, Grasso LC, Chua CM, et al. Whole transcriptome analysis of the coral Acropora millepora reveals complex responses to $\mathrm{CO}(2)$-driven acidification during the initiation of calcification. Mol Ecol. 2012;21(10):2440-54.

115. Lourenco AP, Martins JR, Bitondi MM, Simoes ZL. Trade-off between immune stimulation and expression of storage protein genes. Arch Insect Biochem Physiol. 2009;71(2):70-87.

116. Rauw WM. Immune response from a resource allocation perspective. Front Genet. 2012;3:267.

117. Hegemann A, Matson KD, Versteegh MA, Tieleman BI. Wild Skylarks Seasonally Modulate Energy Budgets but Maintain Energetically Costly Inflammatory Immune Responses throughout the Annual Cycle. PLOS ONE. 2012;7(5):e36358.

118. Simmons LW. Resource allocation trade-off between sperm quality and immunity in the field cricket, Teleogryllus oceanicus. Behav Ecol. 2011;23(1):168-73.

119. Fabbri E, Valbonesi P, Franzellitti S. HSP expression in bivalves. Invertebrate Survival Journal: ISJ. 2008;5:135-61.

120. Moreira R, Balseiro P, Romero A, Dios S, Posada D, Novoa B, et al. Gene expression analysis of clams Ruditapes philippinarum and Ruditapes decussatus following bacterial infection yields molecular insights into pathogen resistance and immunity. Dev Comp Immunol. 2012;36(1):140-9.

121. Genard B, Miner P, Nicolas J-L, Moraga D, Boudry P, Pernet F, et al. Integrative Study of Physiological Changes Associated with Bacterial Infection in Pacific Oyster Larvae. PLoS ONE. 2013;8(5):e64534.

122. Zugel U, Kaufmann SH. Role of heat shock proteins in protection from and pathogenesis of infectious diseases. Clin Microbiol Rev. 1999;12(1):19-39.

123. Osterloh A, Breloer M. Heat shock proteins: linking danger and pathogen recognition. Med Microbiol Immunol. 2008;197(1):1-8.

124. Ouzounis CA, Khatri P, Sirota M, Butte AJ. Ten Years of Pathway Analysis: Current Approaches and Outstanding Challenges. Plos Comput Biol. 2012; 8(2):e1002375.

125. Kamiya H, Muramoto K, Yamazaki M. Aplysianin-A, an antibacterial and antineoplastic glycoprotein in the albumen gland of a sea hare, Aplysia kurodai. Experientia. 1986:42(9):1065-7.
126. lijima R, Kisugi J, Yamazaki M. Antifungal activity of Aplysianin E, a cytotoxic protein of sea hare (Aplysia kurodai) eggs. Dev Comp Immunol. 1995;19(1):13-9.

127. Oliver JL, Gaffney PM, Allen SK, Faisal M, Kaattari SL. Protease Inhibitory Activity in Selectively Bred Families of Eastern Oysters. J Aquat Anim Health 2000;12(2):136-45

128. Chernikov OV, Molchanova VI, Chikalovets IV, Kondrashina AS, Li W, Lukyanov PA. Lectins of marine hydrobionts. Biochemistry (Moscow). 2013;78(7):760-70.

129. Smolowitz R, Defaveri J, Walton W, Murphy D, Leavitt D. The interaction of temperature and hard clam (Mercenaria mercenaria) strain on the occurrence of QPX disease in the laboratory and in the field. J Shellfish Res. 2008;27(4):1052-3

130. Dahl SF, Perrigault M, Allam B. Laboratory transmission studies of QPX disease in the hard clam: Interactions between different host strains and pathogen isolates. Aquaculture. 2008;280(1-4):64-70.

131. Monari M, Foschi J, Rosmini R, Marin MG, Serrazanetti GP. Heat shock protein 70 response to physical and chemical stress in Chamelea gallina. J Exp Mar Biol Ecol. 2011;397(2):71-8.

132. Metzger DC, Pratt $P$, Roberts SB. Characterizing the Effects of Heavy Metal and Vibrio Exposure on Hsp70 Expression in Crassostrea Gigas Gill Tissue. J Shellfish Res. 2012;31(3):627-30.

133. Roberts RJ, Agius C, Saliba C, Bossier P, Sung YY. Heat shock proteins (chaperones) in fish and shellfish and their potential role in relation to fish health: a review. J Fish Dis. 2010;33(10):789-801.

134. Narusaka Y, Narusaka M, Seki M, Umezawa T, Ishida J, Nakajima M, et al. Crosstalk in the responses to abiotic and biotic stresses in Arabidopsis: Analysis of gene expression in cytochrome P450 gene superfamily by cDNA microarray. Plant Mol Biol. 2004;55(3):327-42.

135. Cardona P-J, Seifart Gomes C, Izar B, Pazan F, Mohamed W, Mraheil MA, et al. Universal Stress Proteins Are Important for Oxidative and Acid Stress Resistance and Growth of Listeria monocytogenes EGD-e In Vitro and In Vivo. PLoS ONE. 2011;6(9):e24965.

136. Schmieder R, Lim YW, Edwards R. Identification and removal of ribosomal RNA sequences from metatranscriptomes. Bioinformatics. 2012;28(3):433-5.

137. Grabherr MG, Haas BJ, Yassour M, Levin JZ, Thompson DA, Amit I, et al. Full-length transcriptome assembly from RNA-Seq data without a reference genome. Nat Biotechnol. 2011;29(7):644-52

138. Moriya Y, Itoh M, Okuda S, Yoshizawa AC, Kanehisa M. KAAS: an automatic genome annotation and pathway reconstruction server. Nucleic Acids Res. 2007;35(Web Server issue):W182-5.

139. Anders S, Huber W. Differential expression analysis for sequence count data. Genome Biol. 2010;11(10):R106.

\section{Submit your next manuscript to BioMed Central and we will help you at every step:}

- We accept pre-submission inquiries

- Our selector tool helps you to find the most relevant journal

- We provide round the clock customer support

- Convenient online submission

- Thorough peer review

- Inclusion in PubMed and all major indexing services

- Maximum visibility for your research

Submit your manuscript at www.biomedcentral.com/submit
) Biomed Central 